\title{
Weighted kernel operators in variable exponent amalgam spaces
}

\author{
Vakhtang Kokilashvili ${ }^{1,2}$, Alexander Meskhi ${ }^{1,3}$ and Muhammad Asad Zaighum ${ }^{*}$
}

${ }^{\text {*Correspondence: }}$

asadzaighum@gmail.com

${ }^{4}$ Abdus Salam School of

Mathematical Sciences,

GC University, 68-B New Muslim

Town, Lahore, Pakistan

Full list of author information is

available at the end of the article

\begin{abstract}
The paper is devoted to weighted inequalities for positive kernel operators in variable exponent amalgam spaces. In particular, a characterization of a weight $v$ governing the boundedness/compactness of the weighted kernel operators $K_{v}$ and $\mathcal{K}_{v}$, defined on $\mathbb{R}_{+}$and $\mathbb{R}$, respectively, under the log-Hölder continuity condition on exponents of spaces is established. These operators involve, for example, weighted variable parameter fractional integrals. The results are new even for constant exponent amalgam spaces.
\end{abstract}

MSC: 46E30; 47B34

Keywords: variable exponent amalgam spaces; positive kernel operator; boundedness; compactness

\section{Introduction}

In the paper, we derive necessary and sufficient conditions on a weight function $v$ governing the boundedness/compactness of the positive kernel operators

$$
\begin{aligned}
& K_{v} f(x)=v(x) \int_{0}^{x} k(x, t) f(t) d t, \quad x>0, \\
& \left(\mathcal{K}_{v} f\right)(x)=v(x) \int_{-\infty}^{x} k(x, t) f(t) d t, \quad x \in \mathbb{R}
\end{aligned}
$$

in variable exponent amalgam spaces (VEAS) under the log-Hölder continuity condition on exponents of spaces. It should be emphasized that the results are new even for constant exponent amalgam spaces.

Historically, the boundedness problem for the two-weighted Hardy transform $\left(H_{v, w} f\right)(x)=v(x) \int_{0}^{x} f(t) w(t) d t$ from $L^{p(\cdot)}$ to $L^{q(\cdot)}$ was studied in the papers [1, 2] in different terms on weights (see also [3] for related topics). In [1], the authors explored also the compactness problem for $H_{v, w}$. The boundedness for fractional integral operators in (weighted) variable exponent Lebesgue spaces defined on Euclidean spaces was investigated by many authors (see, e.g., the papers [4-14], etc.). The compactness (resp. non-compactness) of fractional and singular integrals in weighted $L^{p(\cdot)}$ spaces was studied in [15]. We refer also to the monograph [16] for related topics.

The space $L^{p(\cdot)}$ is a special case of the Musielak-Orlicz space (see $\left.[17,18]\right)$. The first systematic study of modular spaces is due to Nakano [19].

\section{Springer}

(c) 2013 Kokilashvili et al.; licensee Springer. This is an Open Access article distributed under the terms of the Creative Commons Attribution License (http://creativecommons.org/licenses/by/2.0), which permits unrestricted use, distribution, and reproduction in any medium, provided the original work is properly cited. 
Variable exponent Lebesgue and Sobolev spaces arise, e.g., in the study of mathematical problems related to applications to mechanics of the continuum medium (see $[16,20]$ and references cited therein).

The manuscript consists of four sections. In Section 2, we recall some well-known facts about variable exponent Lebesgue spaces $L^{p(\cdot)}$. In Section 3, we recall the definition, history and some essential properties of amalgam spaces with a constant exponent, and also known results about the boundedness of some integral operators in these spaces; boundedness criteria for the operators $K_{v}$ and $\mathcal{K}_{\nu}$ in VEAS are also established. The compactness of positive kernel operators in VEAS is studied in Section 4.

Throughout the paper, constants (often different constants in the same series of inequalities) will mainly be denoted by $c$ or $C$; by the symbol $p^{\prime}(x)$, we denote the function $\frac{p(x)}{p(x)-1}$, $1<p(x)<\infty$; the relation $a \approx b$ means that there are positive constants $c_{1}$ and $c_{2}$ such that $c_{1} a \leq b \leq c_{2} a$.

\section{Preliminaries}

We begin this section by the definition and essential properties of variable exponent Lebesgue spaces.

Let $E$ be a measurable set in $\mathbb{R}$ with positive measure. We denote

$$
p_{-}(E):=\inf _{E} p, \quad p_{+}(E):=\sup _{E} p
$$

for a measurable function $p$ on $E$. Suppose that $1<p_{-}(E) \leq p_{+}(E)<\infty$. Denote by $\rho$ a weight function on $E$ (i.e., $\rho$ is an almost everywhere positive measurable function). We say that a measurable function $f$ on $E$ belongs to $L_{\rho}^{p(\cdot)}(E)$ (or to $\left.L_{\rho}^{p(x)}(E)\right)$ if

$$
S_{p(\cdot), \rho}(f)=\int_{E}|f(x)|^{p(x)} \rho(x) d x<\infty
$$

It is a Banach space with respect to the norm (see, e.g., [21-24])

$$
\|f\|_{L_{\rho}^{p(\cdot)}(E)}=\inf \left\{\lambda>0: S_{p(\cdot), \rho}(f / \lambda) \leq 1\right\} .
$$

If $\rho \equiv$ const, then we use the symbol $L^{p(\cdot)}(E)$ (resp. $\left.S_{p(\cdot)}\right)$ instead of $L_{\rho}^{p(\cdot)}(E)\left(\right.$ resp. $\left.S_{p(\cdot), \rho}\right)$. It is clear that $\|f\|_{L_{\rho}^{p(\cdot)}(E)}=\left\|f(\cdot) \rho^{1 / p(\cdot)}(\cdot)\right\|_{L^{p(\cdot)}(E)}$.

In the sequel, we will denote by $\mathbb{Z}$ and $\mathbb{Z}_{-}$the set of all integers and the set of non-positive integers, respectively.

To prove the main results, we need some known statements.

Proposition A ([22-24]) Let E be a measurable subset of $\mathbb{R}$. Suppose that $1<p_{-}(E) \leq$ $p_{+}(E)<\infty$. Then

(i)

$$
\begin{array}{ll}
\|f\|_{L^{(\cdot)}(E)}^{p_{+}(E)} \leq S_{p}\left(f \chi_{E}\right) \leq\|f\|_{L^{p(\cdot)}(E)}^{p_{-}(E)}, & \|f\|_{L^{p \cdot(\cdot)(E)}} \leq 1 ; \\
\|f\|_{L^{p(\cdot)}(E)}^{p_{-}(E)} \leq S_{p}\left(f \chi_{E}\right) \leq\|f\|_{L^{p \cdot(\cdot)}(E)^{\prime}}^{p_{+}(E)}, \quad\|f\|_{L^{p(\cdot)}(E)} \geq 1 ;
\end{array}
$$


(ii) Hölder's inequality

$$
\left|\int_{E} f(x) g(x) d x\right| \leq\left(\frac{1}{p_{-}(E)}+\frac{1}{\left(p_{+}(E)\right)^{\prime}}\right)\|f\|_{L^{p(\cdot)}(E)}\|g\|_{L^{p^{\prime} \cdot()(E)}}
$$

holds, where $f \in L^{p(\cdot)}(E), g \in L^{p^{\prime}(\cdot)}(E)$.

Proposition B ([22-24]) Let $1 \leq r(x) \leq p(x)$ and let $E$ be a bounded subset of $\mathbb{R}$. Then the following inequality

$$
\|f\|_{L^{r \cdot(\cdot)}(E)} \leq(|E|+1)\|f\|_{L^{p(\cdot)}(E)}
$$

holds.

Definition 2.1 We say that $p$ satisfies the weak Lipschitz (log-Hölder continuity) condition on $E \subset \mathbb{R}(p \in \mathrm{WL}(E))$, if there is a positive constant $A$ such that for all $x$ and $y$ in $E$ with $0<|x-y|<1 / 2$ the inequality

$$
|p(x)-p(y)| \leq A /(-\ln |x-y|)
$$

holds.

Lemma A ([25]) Let I be an interval in $\mathbb{R}$. Then $p \in \mathrm{WL}(I)$ if and only if there exists a positive constant $c$ such that

$$
|J|^{p_{-}(J)-p_{+}(J)} \leq c
$$

for all intervals $J \subseteq I$ with $|J|>0$. Moreover, the constant $c$ does not depend on $I$.

For the next statement we refer to [2] in the case of finite interval, and [26] for infinite interval.

Proposition C Let $p$ and $q$ be measurable functions on $I:=(a, b)(-\infty<a<b \leq+\infty)$ satisfying the condition $1<p_{-}(I) \leq p(x) \leq q(x)<q_{+}(I)<\infty, x \in I$. Let $p, q \in \mathrm{WL}(I)$. Suppose also that if $b=\infty$, then $p(x) \equiv p_{c} \equiv$ const, $q(x) \equiv q_{c} \equiv$ const outside some large interval $(a, d)$. Then there is a positive constant $c$ depending only on $p$ and $q$ such that for all $f \in L^{p(\cdot)}(I), g \in L^{q^{\prime}(\cdot)}(I)$ and all sequences of intervals $S_{k}:=\left[x_{k-1}, x_{k+1}\right)$, where $\left[x_{k}, x_{k+1}\right)$ are disjoint intervals satisfying the condition $\bigcup_{k}\left[x_{k}, x_{k+1}\right)=I$, the inequality

$$
\sum_{k}\left\|f \chi_{S_{k}}\right\|_{L^{p(\cdot)(I)}}\left\|g \chi_{S_{k}}\right\|_{L^{q^{\prime}(\cdot)(I)}} \leq c C_{a, b}\|f\|_{L^{p(\cdot)}(I)}\|g\|_{L^{q^{\prime} \cdot(\cdot)(I)}}
$$

holds. Moreover, the value of $C_{a, b}$ is defined as follows: $C_{a, b}=[(b-a)+1]^{2}$ if $b<\infty$ and $C_{a, \infty}=[(d-a)+1]^{2}+1$ if $b=\infty$.

Let $v$ and $w$ be a.e. positive measurable function on $[a, b),-\infty<a<b \leq \infty$, and let

$$
\left(H_{v, w}^{(a, b)} f\right)(x)=v(x) \int_{a}^{x} f(t) w(t) d t, \quad x \in[a, b) .
$$


Further, we denote

$$
\begin{aligned}
& \left(H_{v, w} f\right)(x)=v(x) \int_{0}^{x} f(t) w(t) d t, \quad x>0, \\
& \left(\mathcal{H}_{v, w} f\right)(x)=v(x) \int_{-\infty}^{x} f(t) w(t) d t, \quad x \in \mathbb{R} .
\end{aligned}
$$

Let us recall the two-weight criterion for the Hardy operator in classical Lebesgue spaces:

Theorem A $([27,28])$ Let $r$ and $s$ be constants such that $1<r \leq s<\infty$. Suppose that $0 \leq$ $a<b \leq \infty$. Let $v$ and $w$ be non-negative measurable functions on $[a, b)$. Then the Hardy inequality

$$
\left(\int_{a}^{b} v(x)\left(\int_{a}^{x} f(t) d t\right)^{s} d x\right)^{1 / s} \leq c\left(\int_{a}^{b} w(t)(f(t))^{r} d t\right)^{1 / r}, \quad f \geq 0,
$$

holds if and only if

$$
A:=\sup _{a \leq t \leq b}\left(\int_{t}^{b} v(x) d x\right)^{1 / s}\left(\int_{a}^{t} w^{1-r^{\prime}}(x) d x\right)^{1 / r^{\prime}}<\infty .
$$

Moreover, if $c$ is the best constant in the Hardy inequality, then there are positive constants $c_{1}$ and $c_{2}$ depending only on $r$ and s such that $c_{1} A \leq c \leq c_{2} A$.

For the Hardy inequalities, we also refer the books [29, 30].

The following statement was proved in [2] for finite interval and in [12] for the case of infinite interval, but we give the proof because of the upper and lower bound of the norm of $H_{v, w}$.

Theorem B Let $-\infty<a<b \leq+\infty$ and let $p$ and $q$ be measurable functions on $I:=(a, b)$ satisfying the conditions: $1<p_{-}(I) \leq p(x) \leq q(x) \leq q_{+}(I)<\infty, p, q \in \mathrm{WL}(I)$. We assume that $p \equiv p_{c} \equiv$ const, $q \equiv q_{c} \equiv$ const outside some large interval $(a, d)$ if $b=\infty$. Then $H_{v, w}^{I}$ is bounded from $L^{p(\cdot)}(I)$ to $L^{q(\cdot)}(I)$ if and only if

$$
A_{a, b} \equiv \sup _{a<t<b}\left\|\chi_{(t, b)(\cdot)} v(\cdot)\right\|_{L^{q(\cdot)(I)}}\left\|\chi_{(a, t)(\cdot)} w(\cdot)\right\|_{L^{p^{\prime}(\cdot)(I)}}<\infty
$$

Moreover, there are positive constants $c_{1}$ and $c_{2}$ independent of the interval I such that

$$
c_{1} A_{a, b} \leq\left\|H_{v, w}^{(a, b)}\right\|_{L^{p(\cdot)(I) \rightarrow L^{q(\cdot)(I)}}} \leq c_{2} C_{a, b} A_{a, b},
$$

where the constant $C_{a, b}$ is defined in Proposition $\mathrm{C}$.

Proof Sufficiency. Let $f \geq 0$. Suppose that $b<\infty$ and that $\int_{a}^{b} f(t) d t \in\left[2^{m_{0}}, 2^{m_{0}+1}\right)$ for some integer $m_{0}$. We construct a sequence $\left\{x_{k}\right\}$ so that

$$
\int_{a}^{x_{k}} f w=\int_{x_{k}}^{x_{k+1}} f w=2^{k}
$$


It is easy to check that $(a, b)=\bigcup_{k}\left[x_{k}, x_{k+1}\right)$. Let $g$ be a function satisfying the condition $\|g\|_{L^{q^{\prime}(\cdot)([a, b])}} \leq 1$. Applying Hölder's inequality for variable exponent Lebesgue spaces and Proposition $\mathrm{C}$ we have that

$$
\begin{aligned}
\int_{a}^{b}\left(H_{v, w} f\right) g \leq & \sum_{k}\left(\int_{x_{k}}^{x_{k+1}} g v\right)\left(\int_{0}^{x_{k+1}} f w\right) \\
= & 4 \sum_{k}\left(\int_{x_{k}}^{x_{k+1}} g v\right)\left(\int_{x_{k-1}}^{x_{k}} f w\right) \\
\leq & 4 \sum_{k}\left\|\chi_{\left(x_{k}, x_{k+1}\right)}(\cdot) g(\cdot)\right\|_{L^{q^{\prime} \cdot(\cdot)(I)}}\left\|\chi_{\left(x_{k}, x_{k+1}\right)}(\cdot) v(\cdot)\right\|_{L^{q(\cdot)(I)}} \\
& \times\left\|\chi_{\left(x_{k-1}, x_{k}\right)}(\cdot) f(\cdot)\right\|_{L^{p \cdot(\cdot)(I)}}\left\|\chi_{\left(x_{k-1}, x_{k}\right)}(\cdot) w(\cdot)\right\|_{L^{p^{\prime}(\cdot)(I)}} \\
\leq & 4 A_{a, b} \sum_{k}\left\|\chi_{\left(x_{k}, x_{k+1}\right)}(\cdot) g(\cdot)\right\|_{L^{q^{\prime}(\cdot)(I)}}\left\|\chi_{\left(x_{k-1}, x_{k}\right)}(\cdot) f(\cdot)\right\|_{L^{p \cdot(\cdot)(I)}} \\
\leq & 4 C_{a, b} A_{a, b}\|f(\cdot)\|_{L^{p(\cdot)(I)}}\|g(\cdot)\|_{L^{q^{\prime} \cdot(\cdot)(I)}},
\end{aligned}
$$

where $C_{a, b}$ is the constant defined in Proposition C. Taking now the supremum with respect to $g$, we have sufficiency for $b<\infty$.

Let now $b=\infty$. Then

$$
\begin{aligned}
\left\|H_{v, w}^{(a, \infty)} f\right\|_{L^{q(\cdot)}((a,+\infty))} & \leq\left\|v(x) \int_{a}^{x} f w\right\|_{L^{q \cdot \cdot((a, d))}}+\left\|v(x) \int_{a}^{x} f w\right\|_{L^{q^{c}([d,+\infty))}} \\
& :=I_{1}+I_{2} .
\end{aligned}
$$

By applying already used arguments, we have that $I_{1} \leq 4 C_{a, \infty} A_{a,+\infty}$, where $C_{a, \infty}=[(d-$ $a)+1]^{2}$. Further, due to Hölder's inequality and Theorem A, we find that

$$
\begin{aligned}
I_{2} \leq & \left\|v(x) \int_{a}^{d} f w\right\|_{L^{q^{c}([d,+\infty))}}+\left\|v(x) \int_{d}^{x} f w\right\|_{L^{q_{c}([d,+\infty))}} \\
\leq & \left\|v(\cdot) \chi_{[d,+\infty)}(\cdot)\right\|_{L^{q(\cdot)}}\left\|w(\cdot) \chi_{[a, d)}(\cdot)\right\|_{L^{p^{\prime}(\cdot)}}\|f\|_{L^{p(\cdot)}} \\
& +4 A_{a,+\infty}\|f\|_{L^{p(\cdot)}(I)} \leq 5 A_{a,+\infty}\|f\|_{L^{p \cdot(\cdot)}(I)} .
\end{aligned}
$$

To get the lower bound for $\left\|H_{v, w}^{(a, b)}\right\|$ is trivial by choosing the appropriate test function $f(x)=\chi_{(a, t)}(x), a<t<b$ in the boundedness of $H_{v, w}^{I}$ from $L^{p(\cdot)}(I)$ to $L^{q(\cdot)}(I)$.

Corollary A Let $p$ and $q$ be defined on $\mathbb{R}_{+}$and satisfy the conditions of Theorem $\mathrm{B}$. Then for all $n \in \mathbb{Z}$,

$$
\left\|v(x) \int_{2^{n}}^{x} f(t) w(t) d t\right\|_{L^{q(\cdot)\left(\left[2^{n}, 2^{n+1}\right]\right)}} \leq D\|f\|_{L^{p \cdot()\left(\left[2^{n}, 2^{n+1}\right]\right)}}
$$

where $D=\max \left\{c(2 d+1)^{2}, 4\right\} \sup _{n \in \mathbb{Z}} A_{2^{n}, 2^{n+1}}, A_{2^{n}, 2^{n+1}}$ is defined in Theorem $\mathrm{B}$ and the constant $c$ depends only on $p$ and $q$. 
Proof By the hypothesis, $p$ and $q$ are constant outside some large interval $(0, d)$. Let $d \in$ $\left[2^{m_{0}-1}, 2^{m_{0}}\right)$ for some integer $m_{0}$. Then by Theorem B for $n \leq m_{0}$, we have

$$
\begin{aligned}
\left\|H_{v, w}^{\left(2^{n}, 2^{n+1}\right)}\right\|_{L^{p \cdot(\cdot)}\left(\left[2^{n}, 2^{n+1}\right)\right) \rightarrow L^{q \cdot \cdot\left(\cdot\left(2^{n}, 2^{n+1}\right)\right)}} \leq c\left(2^{n}+1\right)^{2} A_{2^{n}, 2^{n+1}} \\
\leq c\left(2^{m_{0}}+1\right)^{2} A_{2^{n}, 2^{n+1}} \\
\leq c(2 d+1)^{2} \sup _{n \in \mathbb{Z}} A_{2^{n}, 2^{n+1}}
\end{aligned}
$$

where the positive constant $c$ depends only on $p$ and $q$. If $n>m_{0}$, then $p$ and $q$ are constants on the intervals $\left[2^{n}, 2^{n+1}\right)$. In this case taking the proof of Theorem B into account, we find that

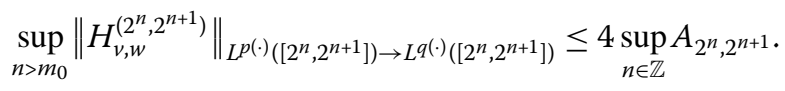

Theorem C ([5]) Let $p(x)$ and $q(x)$ be measurable functions on an interval $I \subseteq R_{+}$. Suppose that $1<p_{-}(I) \leq p_{+}(I)<\infty$ and $1<q_{-}(I) \leq q_{+}(I)<\infty$. If

$$
\|\| k(x, y)\left\|_{L^{p^{\prime}(y)}(I)}\right\|_{L^{q(x)}(I)}<\infty
$$

where $k$ is a non-negative kernel, then the operator

$$
K f(x)=\int_{I} k(x, y) f(y) d y
$$

is compact from $L^{p(\cdot)}(I)$ to $L^{q(\cdot)}(I)$.

Lemma B (see, e.g. [31]) Let $1<q<\bar{q}<\infty$ and $\frac{1}{s}=\frac{1}{q}-\frac{1}{\bar{q}}$. Suppose that $\left\{u_{n}\right\}$ and $\left\{v_{n}\right\}$ are sequences of positive real numbers. The following statements are equivalent:

(i) There exists $C>0$ such that the inequality

$$
\left\{\sum_{n \in \mathbb{Z}}\left(\left|a_{n}\right| u_{n}\right)^{q}\right\}^{1 / q} \leq C\left\{\sum_{n \in \mathbb{Z}}\left(\left|a_{n}\right| v_{n}\right)^{\bar{q}}\right\}^{1 / \bar{q}}
$$

holds for all sequences $\left\{a_{n}\right\}$ of real numbers.

(ii) $\left\{\sum_{n \in \mathbb{Z}}\left(u_{n} v_{n}^{-1}\right)^{s}\right\}^{1 / s}<\infty$.

Lemma C (see e.g. [32]) Let $p, q$ be constants such that $1<p, q<\infty$. Suppose that $v_{k} \geq 0$, $w_{k}>0, k \in \mathbb{Z}$. Then there exists a constant $c>0$ such that

$$
\left\{\sum_{n \in \mathbb{Z}}\left(\sum_{k=-\infty}^{n} v_{n} a_{k}\right)^{q}\right\}^{1 / q} \leq c\left(\sum_{n \in \mathbb{Z}}\left(w_{n} a_{n}\right)^{p}\right)^{1 / p}
$$

holds for all non-negative sequence $\left\{a_{k}\right\} \in l_{\left\{v_{n}^{p}\right.}^{p}$, if and only if

(i) in case $1<p \leq q<\infty$,

$$
A_{1}:=\sup _{m \in \mathbb{Z}}\left(\sum_{n=m}^{\infty} v_{n}^{q}\right)^{1 / q}\left(\sum_{n=-\infty}^{m} w_{n}^{-p^{\prime}}\right)^{1 / p^{\prime}}<\infty ;
$$


(ii) in case $1<q<p<\infty$,

$$
A_{2}:=\left\{\sum_{m \in \mathbb{Z}}\left(\sum_{n=m}^{\infty} v_{n}^{q}\right)^{r / q}\left(\sum_{n=-\infty}^{m} w_{n}^{-p^{\prime}}\right)^{r / q^{\prime}} w_{m}^{-p^{\prime}}\right\}^{1 / r}<\infty,
$$

where $1 / r=1 / q-1 / p$.

Definition 2.2 Let $I=(0, a), 0<a \leq \infty$. We say that a kernel $k:\{(x, y): 0<y<x<a\} \rightarrow$ $(0, \infty)$ belongs to $V(I)(k \in V(I))$ if there exists a constant $c_{1}$ such that for all $x, y, t$ with $0<y<t<x<a$ the inequality

$$
k(x, y) \leq c_{1} k(x, t)
$$

holds.

Definition 2.3 Let $r$ be a measurable function on $I=(0, a), 0<a \leq \infty$ with values in $(1,+\infty)$. We say a kernel $k$ belongs to $V_{r(\cdot)}(I)$ if there exists a positive constant $c_{2}$ such that for a.e. $x \in(0, a)$, the inequality

$$
\left\|\chi\left(\frac{x}{2}, x\right)(\cdot) k(x, \cdot)\right\|_{L^{r(\cdot)(I)}} \leq c_{2} x^{\frac{1}{r(x)}} k\left(x, \frac{x}{2}\right)
$$

is fulfilled.

Example 2.1 (Lemma 3 of [26]) Let $I:=(0, a)$, where $0<a \leq \infty$. Let $\alpha$ be a measurable function on $I$ satisfying the condition $0<\alpha_{-}(I) \leq \alpha_{+}(I) \leq 1$. Suppose that $r$ is a function on $I$ with values in $(1,+\infty)$ satisfying the condition $r \in \mathrm{WL}(I)$. Suppose that $r(x) \equiv r_{0} \equiv$ const outside some interval $(0, b)$ when $a=+\infty$. Then $k(x, t)=(x-t)^{\alpha(x)-1} \in V(I) \cap V_{r(\cdot)}(I)$ when $r(x)<\frac{1}{1-\alpha(x)}$.

The next examples of kernels can be checked easily:

Example 2.2 Let $I:=(0, a)$, where $0<a \leq \infty$. Suppose that $\alpha$ is a measurable function on $I$ satisfying the condition $0<\alpha_{-}(I) \leq \alpha_{+}(I) \leq 1$. Let $r$ be a function on $I$ with the values in $(1,+\infty)$ satisfying the condition $r, \bar{r} \in \mathrm{WL}(I)$ where $\bar{r}(t)=r\left(t^{1 / \sigma}\right)$. Suppose that $r(x) \equiv r_{0} \equiv$ const outside some interval $(0, b)$ when $a=+\infty$. Then $k(x, y)=\left(x^{\sigma}-y^{\sigma}\right)^{\alpha(x)-1} \in V(I) \cap$ $V_{r(\cdot)}(I)$ when $r(x)<\frac{1}{1-\alpha(x)}$ and $\sigma>0$.

Example 2.3 Let $I:=(0, a), 0<a \leq \infty$. Let $r$ be a function on $I$ with the values in $(1,+\infty)$ satisfying the condition $r \in \mathrm{WL}(I)$ and let $r$ be increasing on $I$. Suppose that $r(x) \equiv r_{0} \equiv$ const outside some interval $(0, b)$ when $a=+\infty$. Further, let $0<\alpha_{-}(I) \leq \alpha(x) \leq 1$ and $\alpha(x)+$ $\beta(x)>2-\frac{1}{r(x)}$. Then $k(x, y)=(x-y)^{\alpha(x)-1} \ln ^{\beta(x)-1} \frac{x}{y} \in V(I) \cap V_{r(\cdot)}(I)$.

For other examples of kernel $k$ satisfying the condition $k \in V(I) \cap V_{r}(I)$, where $r$ is constant, we refer to [33] (see also [34], p.163).

\section{Boundedness on VEAS}

This section is devoted to the boundedness of weighted kernel operators in VEAS. 


\subsection{Amalgam spaces}

Let $I$ be $\mathbb{R}$ or $\mathbb{R}_{+}$and $\alpha=\left\{I_{n} ; n \in \mathbb{Z}\right\}$ be a cover of $I$ consisting of disjoint half-open intervals $I_{n}$, each of the form $\left[a_{1}, a_{2}\right)$, whose union is $I$. Let

$$
\|f\|_{\left(L_{u}^{p(\cdot)}(I), l^{q}\right)_{\alpha}}:=\left(\sum_{n \in \mathbb{Z}}\left\|\chi_{I_{n}}(\cdot) f(\cdot)\right\|_{L_{u}^{p(\cdot)}(I)}^{q}\right)^{1 / q},
$$

we define the general amalgams with variable exponent

$$
\left(L_{u}^{p(\cdot)}(I), l^{q}\right)_{\alpha}=\left\{f:\|f\|_{\left(L_{u}^{p(\cdot)}(I), l^{q}\right)_{\alpha}}<\infty\right\}
$$

If $u \equiv$ const, then $\left(L_{u}^{p(\cdot)}(I), l^{q}\right)_{\alpha}$ is denoted by $\left(L^{p(\cdot)}(I), l^{q}\right)_{\alpha}$.

Let $p \equiv p_{c} \equiv$ const and $u \equiv$ const. Then we have the usual irregular amalgam (see [35]); if $I=\mathbb{R}$ and $I_{n}=[n, n+1)$, then $\left(L^{p_{c}}(I), l^{q}\right)_{\alpha}$ is the amalgam space introduced by Wiener (see $[36,37])$ in connection with the development of the theory of generalized harmonic analysis.

We call $\left(L_{u}^{p(\cdot)}(I), l^{q}\right)_{\alpha}$ irregular weighted amalgam spaces with variable exponent. If $I_{n}=$ $[n, n+1)$, then $\left(L_{u}^{p(\cdot)}(I), l^{q}\right)_{\alpha}$ will be denoted by $\left(L_{u}^{p(\cdot)}(I), l^{q}\right)$.

Let $d=\left\{\left[2^{n}, 2^{n+1}\right) ; n \in \mathbb{Z}\right\}$ and $I=\mathbb{R}_{+}$. We denote weighted dyadic amalgam with variable exponent by $\left(L_{u}^{p(\cdot)}(I), l^{q}\right)_{d}$. Some properties regarding general amalgams with variable exponent can be derived in the same way as for usual irregular amalgams $\left(L_{u}^{p}(\mathbb{R}), l^{q}\right)_{\alpha}$, where $p$ is constant. Irregular amalgams were introduced in [38] and studied in [35].

Theorem D Let $p$ be a measurable function on $I$ with $1<p_{-}(I) \leq p_{+}(I)<\infty$ and $q$ be constant with $1<q<\infty$. The irregular amalgams with variable exponent $\left(L^{p(\cdot)}(I), l^{q}\right)_{\alpha}$ is a Banach space whose dual space is $\left(L^{p(\cdot)}(I), l^{q}\right)_{\alpha}^{*}=\left(L^{p^{\prime}(\cdot)}(I), l^{q^{\prime}}\right)_{\alpha}$. Further, Hölder's inequality holds in the following form:

$$
\left|\int_{I} f(t) g(t) d t\right| \leq\|f\|_{\left(L^{p(\cdot)(I), l q}\right)_{\alpha}}\|g\|_{\left(L^{\left.(p(\cdot))^{\prime}(I), l^{\prime}\right)_{\alpha}}\right.} .
$$

Proof Since $L^{p(\cdot)}$ is a Banach space and $\left(L^{p(\cdot)}\right)^{*}=L^{p^{\prime}(\cdot)}$ (see [22]), from general arguments (see [35, 39-41]) we have the desired result.

The next statement for more general case, i.e., when amalgams are defined with respect to Banach spaces, can be found in [35].

Theorem E Let $p$ be measurable function on I and $1 \leq q_{1} \leq q_{2}$, then

$$
\left(L^{p(\cdot)}(I), l^{q_{1}}\right)_{\alpha} \subset\left(L^{p(\cdot)}(I), l^{q_{2}}\right)_{\alpha}
$$

Other structural properties of amalgams are investigated, e.g., in [41] and [35].

The next statement is a generalization of Theorem 4 in [35] for variable exponent amalgams with weights.

Proposition D Let $p, q$ be measurable functions on I such that $1 \leq q_{-}(I) \leq q(x)<p(x) \leq$ $p_{+}(I)$ and $1 \leq r<\infty$. Then the space $\left(L_{w}^{p(\cdot)}(I), l^{r}\right)_{\alpha}$ is continuously embedded in $\left(L_{v}^{q(\cdot)}(I), l^{r}\right)_{\alpha}$ 


$$
S:=\sup _{n \in \mathbb{Z}} \int_{I_{n}}\left(\frac{v(x)}{w(x)}\right)^{\frac{p(x)}{p(x)-q(x)}} d x<\infty .
$$

Conversely, if $1<q_{-}(I) \leq q_{+}(I)<p_{-}(I) \leq p_{+}(I)<\infty$, then condition (3.1) is also necessary for the continuous embedding of $\left(L_{w}^{p(\cdot)}(I), l^{r}\right)_{\alpha}$ into $\left(L_{v}^{q(\cdot)}(I), l^{r}\right)_{\alpha}$.

Proof It is known (see [42]) that the continuous embedding $L_{w}^{p(\cdot)}(I) \hookrightarrow L_{v}^{q(\cdot)}(I)(q(x)<p(x))$ holds if and only if

$$
\int_{I}\left(\frac{v(x)}{w(x)}\right)^{\frac{p(x)}{p(x)-q(x)}} d x<\infty
$$

Moreover, the estimate

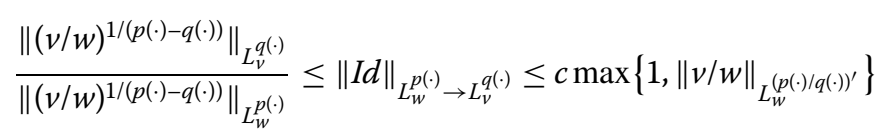

holds, where the positive constant $c$ depends only on $p$ and $q$; Id is the identity operator. Let condition (3.1) hold. Then

$$
\|I d\|_{L_{w}^{p(\cdot)}\left(I_{n}\right) \rightarrow L_{v}^{q \cdot \cdot\left(I_{n}\right)}} \leq\|I d\|_{L_{w}^{p(\cdot)}(I) \rightarrow L_{v}^{q(\cdot)}(I)}<\infty .
$$

Hence, $\left(L^{p(\cdot)}, l^{r}\right)_{\alpha} \hookrightarrow\left(L^{q(\cdot)}, l^{r}\right)_{\alpha}$.

Conversely, let the continuous embedding $\left(L^{p(\cdot)}, l^{r}\right)_{\alpha} \hookrightarrow\left(L^{q(\cdot)}, l^{r}\right)_{\alpha}$ hold and let $1<q_{-}(I) \leq$ $q_{+}(I)<p_{-}(I) \leq p_{+}(I)<\infty$. By taking functions supported in $I_{n}$ we can derive the estimate

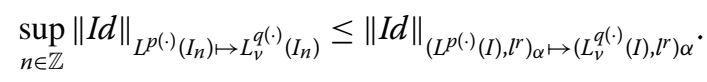

By applying the left-hand side inequality of $\left(3.1^{\prime}\right)$ and Proposition A, we conclude that condition (3.1) is satisfied.

\subsection{General operators in VEAS}

We begin this subsection by the following definition.

Definition 3.1 ([31]) Let $T$ be an operator defined on a set of real measurable functions $f$ on $\mathbb{R}$. Define a sequence of local operators

$$
\left(T_{n} f\right)(x):=T\left(f \chi_{(n-1, n+2)}\right)(x), \quad x \in(n-1, n+2), n \in \mathbb{Z} .
$$

Let us assume that there is a discrete operator $T^{d}$ satisfying the following conditions:

(i) There exists a positive constant $c$ such that for all non-negative functions $f$, $x \in(n, n+1)$ and arbitrary $n \in \mathbb{Z}$ the inequality

$$
T\left(f \chi_{(-\infty, n-1)}+f \chi_{(n+2, \infty)}\right)(x) \leq c T^{d}\left(\int_{m-1}^{m} f\right)(n)
$$

holds. 
(ii) There is $c>0$ such that for all sequences $\left\{a_{k}\right\}$ of non-negative real numbers and $n \in \mathbb{Z}$, the inequality

$$
T^{d}\left(\left\{a_{k}\right\}\right)(n) \leq c T f(y)
$$

holds for all $y \in(n, n+1)$ and all non-negative $f$, where $\int_{m-1}^{m} f=: a_{m}, m \in \mathbb{Z}$. It is also assumed that $T$ satisfies the conditions

$$
T f=T|f|, \quad T(\lambda f)=|\lambda| T f, \quad T(f+g) \leq T f+T g, \quad T f \leq T g \quad \text { if } f \leq g .
$$

We will say that an operator $T$ satisfying all the above mentioned conditions is admissible on $\mathbb{R}$.

For example, Hardy operators, Hardy-Littlewood maximal operators, fractional integral operators, fractional maximal operators are admissible on $\mathbb{R}$ (see [31]). Carton-Leburn, Heinig and Hoffmann [32] established two weighted criteria for the Hardy transform $(\mathcal{H} f)(x)=\int_{-\infty}^{x} f(t) d t$ in amalgam spaces defined on $\mathbb{R}$ (see also $[43,44]$ for related topics). In [32], the authors derived some sufficient conditions for the two-weight boundedness of the kernel operator $(\mathcal{K} f)(x):=\int_{-\infty}^{x} k(x, y) f(y) d y$ where $k$ is non-decreasing in the second variable and non-increasing in the first one. In the paper [45], the two-weight problem for generalized Hardy-type kernel operators including the fractional integrals of order greater than one (without singularity) was solved.

General type results for the admissible operators read as follows.

Theorem $\mathbf{F}([31])$ Let $1<p, \bar{p}, q, \bar{q}<\infty$, and let $w$ and $v$ be weight functions on $\mathbb{R}$. Suppose that $T$ is an admissible operator on $\mathbb{R}$. Then the inequality

$$
\|v T f\|_{\left(L^{p}(\mathbb{R}), l q\right)} \leq c\|w f\|_{\left(L^{\bar{p}}(\mathbb{R}), l \bar{q}\right)}
$$

\section{holds for all measurable $f$ if and only if}

(i) $T^{d}$ is bounded from $l^{\bar{q}}\left(\left\{w_{n}\right\}\right)$ to $l^{q}\left(\left\{v_{n}\right\}\right)$, where $w_{n}:=\left(\int_{n-1}^{n} w^{-\bar{p}^{\prime}}\right)^{\frac{-\bar{q}}{\bar{p}^{\prime}}}, v_{n}:=\left(\int_{n}^{n+1} v^{p}\right)^{\frac{q}{p}}$.

(ii) (a) $\sup _{n \in \mathbb{Z}}\left\|T_{n}\right\|_{\left[L_{w \bar{p}}^{\bar{p}}(n-1, n+2) \rightarrow L_{v p}^{p}(n-1, n+2)\right]}<\infty$ for $1<\bar{q} \leq q<\infty$.

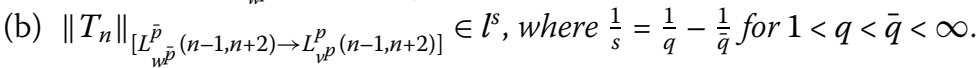

Our aim is to establish weighted characterization of the boundedness of kernel operators involving fractional integrals of variable parameter of order less than one in variable exponent amalgam spaces. For the continuous part of amalgam spaces, we take variable exponent Lebesgue spaces defined on $I$.

It should be emphasized that the following fact holds: by the change of variable $z \rightarrow$ $\log _{2} x$ it is possible to get appropriate boundedness or compactness results from dyadic amalgams $\left(L^{p(\cdot)}\left(\mathbb{R}_{+}\right), l^{q}\right)_{d}$ to amalgams defined on $\mathbb{R}$.

Analyzing the proof of Theorem 1 of [31], we can formulate the next statement and give the proof for completeness.

Proposition 3.1 Let $\bar{p}(\cdot), p(\cdot)$ be measurable functions on $\mathbb{R}$ satisfying $1<p_{-}(\mathbb{R}) \leq p_{+}(\mathbb{R})<$ $\infty, 1<\bar{p}_{-}(\mathbb{R}) \leq \bar{p}_{+}(\mathbb{R})<\infty$. Suppose that $q$ and $\bar{q}$ are constants satisfying $1<q, \bar{q}<\infty$. 
Assume that $w$ and $v$ are weight functions on $\mathbb{R}$ and that $T$ is an admissible operator on $\mathbb{R}$.

Then the inequality

$$
\|v T f\|_{\left(L^{p \cdot \cdot(}(\mathbb{R}), l^{q}\right)} \leq c\|w f\|_{\left(L^{\bar{p} \cdot(\cdot)}(\mathbb{R}), l^{\bar{q}}\right)}
$$

holds if

(i) $T^{d}$ is bounded from $l^{\bar{q}}\left(\left\{\bar{w}_{n}\right\}\right)$ to $l^{q}\left(\left\{\bar{v}_{n}\right\}\right)$ where $\bar{w}_{n}:=\left\|\chi_{(n-1, n)}(\cdot) w^{-1}(\cdot)\right\|_{L^{\bar{p}^{\prime}(\cdot)}}^{-\bar{q}}$, $\bar{v}_{n}:=\left\|\chi_{(n, n+1)}(\cdot) \nu(\cdot)\right\|_{L^{p(\cdot)}}^{q}$.

(ii) (a) $\sup _{n \in \mathbb{Z}}\left\|T_{n}\right\|_{\left[L_{w^{p}(\cdot)}^{\bar{p}(\cdot)}(n-1, n+2) \rightarrow L_{p^{p}(\cdot)}^{p(\cdot)}(n-1, n+2)\right]}<\infty$ for $1<\bar{q} \leq q<\infty$.

(b) $\left\|T_{n}\right\|_{\left[L_{w}^{\bar{p} \cdot(\cdot)}(n-1, n+2) \rightarrow L_{v}^{p \cdot(\cdot)}(n-1, n+2)\right]} \in l^{s}$ with $\frac{1}{s}=\frac{1}{q}-\frac{1}{\bar{q}}$ for $1<q<\bar{q}<\infty$.

Conversely, let (3.2) hold. Then

(1) conditions (ii) are satisfied;

(2) condition (i) is satisfied for $w \equiv$ const or for $p$ and $\bar{p}$ being constants outside some large interval $\left[-m_{0}, m_{0}\right], m_{0} \in \mathbb{Z}$.

Proof Let (i) and (ii) hold. We have

$$
\begin{aligned}
\|v T f\|_{\left(L^{p(\cdot)}(\mathbb{R}), l^{q}\right)} \leq & c\left\{\sum_{n \in \mathbb{Z}}\left\|T\left[f\left(\chi_{(-\infty, n-1)}+\chi_{(n+2, \infty)}\right)\right] v(\cdot)\right\|_{L^{p \cdot()}(n, n+1)}^{q}\right\}^{1 / q} \\
& +c\left\{\sum_{n \in \mathbb{Z}}\left\|v T_{n} f\right\|_{L^{p \cdot(\cdot)}(n, n+1)}\right\}^{1 / q}=: S_{1}+S_{2} .
\end{aligned}
$$

Let $a_{m}:=\int_{m-1}^{m} f$. By the hypothesis and Hölder's inequality for variable exponents $p(\cdot)$ and $p^{\prime}(\cdot)$, we have that

$$
\begin{aligned}
& S_{1} \leq c\left\{\sum_{n \in \mathbb{Z}}\left(T^{d}\left(\left\{a_{m}\right\}\right)(n)\right)^{q}\left\|\chi_{(n, n+1)} v\right\|_{L^{p(\cdot)}(n, n+1)}^{q}\right\}^{1 / q} \leq c\left\{\sum_{n \in \mathbb{Z}} a_{n}^{\bar{q}}\left\|\chi_{(n-1, n)} w^{-1}\right\|_{L^{\bar{p}^{\prime} \cdot(\cdot)}}^{-\bar{q}}\right\}^{1 / \bar{q}} \\
& \leq c\|w f\|_{\left(L^{(\cdot)}\left(\mathbb{R}_{)}, l^{q}\right)\right.} .
\end{aligned}
$$

Let us estimate $S_{2}$. Suppose that $1<\bar{q} \leq q<\infty$. Since the operators $T_{n}$ are uniformly bounded, we find that

$$
\begin{aligned}
S_{2} & \leq c\left\{\sum_{n \in \mathbb{Z}}\|f w\|_{L^{\bar{p} \cdot(\cdot)}(n-1, n+1)}^{q}\right\}^{1 / q} \leq c\left\{\sum_{n \in \mathbb{Z}}\|f w\|_{L^{\bar{p} \cdot(\cdot)}(n-1, n+1)}^{\overline{\bar{q}}}\right\}^{1 / \bar{q}} \\
& \leq c\|f w\|_{\left(L^{\bar{p} \cdot(\cdot)}(\mathbb{R}), \bar{l}^{\bar{q}}\right)} .
\end{aligned}
$$

If $1<q<\bar{q}<\infty$, then by using Hölder's inequality we derive

$$
\begin{aligned}
S_{2} & \leq c\left\{\sum_{n \in \mathbb{Z}}\left\|T_{n}\right\|_{\left[L_{w^{p}}^{\bar{p}(\cdot)}(n-1, n+2) \rightarrow L_{v^{p}}^{p(\cdot)}(n-1, n+2)\right]}^{q}\left\|\chi_{(n-1, n+2)} f w\right\|_{L^{\bar{p} \cdot(\cdot)}}^{q}\right\}^{1 / q} \\
& \leq c\left[\left\{\sum_{n \in \mathbb{Z}}\left\|T_{n}\right\|^{\frac{q \bar{q}}{q-q}}\right\}^{\frac{\bar{q}-q}{q}}\left\{\sum_{n \in \mathbb{Z}}\left\|\chi_{(n-1, n+2)} f w\right\|_{L^{\bar{p}(\cdot)}}^{\bar{q}}\right\}^{\frac{q}{q}}\right]^{1 / q} \\
& \leq c\|f w\|_{\left(L^{\bar{p}(\cdot)}(\mathbb{R}), \bar{q}^{\bar{q}}\right)} .
\end{aligned}
$$


Conversely, suppose that (3.2) holds. Let $n \in \mathbb{Z}$ and let $f$ be a non-negative function supported in $(n-1, n+2)$. Then

$$
\|f w\|_{\left(L^{\bar{p}} \cdot(\cdot)(\mathbb{R}), l \bar{q}\right)} \leq 3\left\|f w \chi_{(n-1, n+2)}\right\|_{\left(L^{\bar{p}(\cdot)}(\mathbb{R})\right)} \cdot
$$

On the other hand,

$$
\begin{aligned}
\|v T f\|_{\left(L^{p(\cdot), l q)}\right.} & \geq\left\|v \chi_{(n-1, n+2)} T f\right\|_{L^{p \cdot(\cdot)}} \\
& \geq\left\|v \chi_{(n-1, n+2)} T_{n} f\right\|_{L^{p \cdot(\cdot)}} \\
& =\left\|v T_{n} f\right\|_{L^{p \cdot(\cdot)}} .
\end{aligned}
$$

Now due to inequality (3.2), we conclude that (a) of (ii) holds. Let us now show that if $1<q<\bar{q}<\infty$, then (b) of (ii) is satisfied.

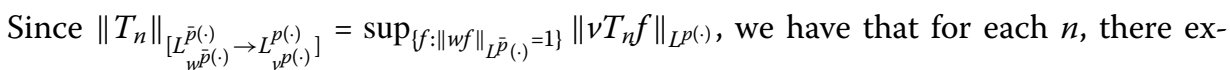
ists a non-negative measurable function $f_{n}$, with the support in $(n-1, n+2)$ and with $\left\|w \chi_{(n-1, n+2)} f_{n}\right\|_{\left.L^{p^{(\cdot)}}\right)}=1$, such that $\left\|T_{n}\right\|_{\left[L_{w^{\bar{p}(\cdot)}}^{\bar{p}^{(\cdot)}} \rightarrow L_{\nu^{p}(\cdot)}^{p^{(\cdot)}}\right]}<\left\|v T_{n} f_{n}\right\|_{L^{p(\cdot)}}+\frac{1}{2^{|n|}}$. Thus, it is sufficient to prove that $\left\|v T_{n} f_{n}\right\|_{L^{p()}} \in l^{s}$.

Let $\left\{a_{n}\right\}$ be a sequence of non-negative real numbers and $f=\sum_{n} a_{n} f_{n}$. For each $n \in \mathbb{Z}$, $f(x)>a_{n} f_{n}(x)$ and then $T f(x) \geq a_{n} T_{n} f_{n}(x)$ for all $x \in(n-1, n+2)$.

Consequently,

$$
\|v T f\|_{\left(L^{p \cdot(\cdot)(\mathbb{R}), l q)}\right.} \geq\left\{\sum_{n \in \mathbb{Z}} c a_{n}^{q}\left\|\chi_{(n-1, n+2)} v T_{n} f\right\|_{L^{p(\cdot)}}^{q}\right\}^{1 / q}=c\left\{\sum_{n \in \mathbb{Z}} a_{n}^{q}\left\|v T_{n} f_{n}\right\|_{L^{p(\cdot)}}^{q}\right\}^{1 / q} .
$$

Hence, inequality (3.2) yields that

$$
\begin{aligned}
\left\{\sum_{n \in \mathbb{Z}} a_{n}^{q}\left\|v T_{n} f_{n}\right\|_{L^{p \cdot(\cdot)}}^{q}\right\}^{1 / q} & \leq c\left\{\sum_{n \in \mathbb{Z}}\left\|\chi_{(n-1, n+2)} w f\right\|_{L^{\bar{p}(\cdot)}}^{\bar{q}}\right\}^{1 / \bar{q}} \\
& \leq c\left\{\sum_{n \in \mathbb{Z}} a_{n}^{\bar{q}}\left\|\chi_{(n-1, n+2)} w f_{n}\right\|_{L^{\bar{p}(\cdot)}}^{\bar{q}}\right\}^{1 / \bar{q}}=c\left\{\sum_{n \in \mathbb{Z}} a_{n}^{\bar{q}}\right\} .
\end{aligned}
$$

Finally, by Lemma B, we see that (b) of (ii) holds.

Now let us prove that (i) holds when $w \equiv$ const. If $\left\{a_{m}\right\}$ is a sequence of non-negative real numbers and

$$
f=\sum_{m \in \mathbb{Z}} a_{m} \chi_{(m-1, m)}
$$

then $\int_{m-1}^{m} f=a_{m}$, and $\left\|\chi_{(n, n+1)} f\right\|_{L^{\bar{p}(\cdot)}}^{\overline{\bar{q}}}=a_{n}^{\bar{q}}\left\|\chi_{(n, n+1)}\right\|_{L^{\bar{p}(\cdot)}}^{\overline{\bar{q}}}=a_{n}^{\bar{q}}$ and by the properties of $T$, we have

$$
\begin{aligned}
\|v T f\|_{\left(L^{\left.p \cdot(\cdot), l^{q}\right)}\right.} & =\left\{\sum_{n \in \mathbb{Z}}\left\|\chi_{(n, n+1)} v T f\right\|_{L^{p \cdot \cdot}}^{q}\right\}^{1 / q} \\
& \geq\left\{\sum_{n \in \mathbb{Z}}\left\|\chi_{(n, n+1)} v T^{d}\left(\int_{m-1}^{m} f\right)\right\|_{L^{p(\cdot)}}^{q}\right\}^{1 / q}
\end{aligned}
$$




$$
\begin{aligned}
& \geq c\left\{\sum_{n \in \mathbb{Z}} T^{d}\left(a_{m}\right)^{q}(n)\left\|\chi_{(n, n+1)} v\right\|_{L^{p \cdot(\cdot)}}^{q}\right\}^{1 / q} \\
& =\left\|T^{d}\left\{a_{m}\right\}\right\|_{l^{q}\left\{\bar{v}_{n}^{q}\right\}} .
\end{aligned}
$$

Applying the two-weight inequality, we find that

$$
\begin{aligned}
\left\|T^{d}\left\{a_{m}\right\}\right\|_{\left.l q_{\left\{\bar{v}_{n}\right.}\right\}} & \leq c\left\{\sum_{n \in \mathbb{Z}}\left\|\chi_{(n, n+1)} f\right\|_{L^{\bar{p}(\cdot)}}^{\bar{q}}\right\}^{1 / \bar{q}} \\
& =c\left\{\sum_{n \in \mathbb{Z}} a_{n}^{\bar{q}}\right\}^{1 / \bar{q}}=\left\|a_{n}\right\|_{l^{\bar{q}} .}
\end{aligned}
$$

Hence, (i) holds.

Suppose now that $w$ is a general weight and there is a positive integer $m_{0}$ such that $p, \bar{p}$ are constants outside $\left[-m_{0}, m_{0}\right]$. Taking

$$
f(x)=\sum_{m \in \mathbb{Z}} a_{m} \chi_{(m-1, m)}(x)\left(\int_{m-1}^{m} w^{-\bar{p}^{\prime}(y)}(y) d y\right)^{-1} w^{-\bar{p}^{\prime}(x)}(x),
$$

it is easy to see that $\int_{m-1}^{m} f=a_{m}$. Moreover, by virtue of Proposition A and the fact that

$$
\int_{m-1}^{m} w^{-\bar{p}^{\prime}(y)}(y) d y \leq \int_{-m_{0}}^{m_{0}} w^{-\bar{p}^{\prime}(y)}(y) d y<\infty, \quad[m-1, m] \subset\left[-m_{0}, m_{0}\right],
$$

we have for $m \leq m_{0}+1$,

$$
\begin{aligned}
\left\|\chi_{(m-1, m)} f w\right\|_{L^{\left.\bar{p}^{(} \cdot\right)}} & =a_{m}\left(\int_{m-1}^{m} w^{-\bar{p}^{\prime}(y)}(y) d y\right)^{-1}\left\|\chi_{(m-1, m)} w^{\left(1-\bar{p}^{\prime}(\cdot)\right)}\right\|_{L^{\left.\bar{p}^{(} \cdot\right)}} \\
& \leq c a_{m}\left(\int_{m-1}^{m} w^{-\bar{p}^{\prime}(y)}(y) d y\right)^{-1 / \bar{p}_{+}([m-1, m))}
\end{aligned}
$$

where the positive constant $c$ depends on $m_{0}$. Since

$$
\|v T f\|_{\left(L^{p \cdot \cdot(\mathbb{R}), l q)}\right.} \geq C\left\|\bar{v}_{n}\left(T^{d}\left\{a_{m}\right\}\right)(n)\right\|_{l q},
$$

using again Proposition A, we find that

$$
\begin{aligned}
\left\|\bar{v}_{n}\left(T^{d}\left\{a_{m}\right\}\right)(n)\right\|_{l q} & \leq C\left[\sum_{m}\left\|\chi_{(m-1, m)} f w\right\|_{L^{\bar{p}(\cdot)(\mathbb{R})}}^{\bar{q}}\right]^{1 / \bar{q}} \\
& \leq c\left[\sum_{m} a_{m}^{\bar{q}}\left(\int_{m-1}^{m} w^{-\bar{p}^{\prime}(y)}(y) d y\right)^{-\bar{q} / \bar{p}_{+}([m-1, m))}\right]^{1 / \bar{q}}=\left\|a_{m} \bar{w}_{m}\right\|_{\bar{q}_{\bar{q}}}
\end{aligned}
$$

Definition 3.2 Let $T$ be an operator defined on a set of real measurable functions $f$ on $\mathbb{R}_{+}$. We say that an operator $T$ is admissible on $\mathbb{R}_{+}$if the conditions of Definition 3.1 are satisfied replacing $n$ by $2^{n}, n \in \mathbb{Z}$.

The next statement can be obtained in the similar manner as Proposition 3.1 was proved; therefore, we omit the proof. 
Proposition 3.2 Let $\bar{p}(\cdot)$, $p(\cdot)$ be measurable functions on $\mathbb{R}_{+}$satisfying $1<p_{-}\left(\mathbb{R}_{+}\right) \leq$ $p_{+}\left(\mathbb{R}_{+}\right)<\infty, 1<\bar{p}_{-}\left(\mathbb{R}_{+}\right) \leq \bar{p}_{+}\left(\mathbb{R}_{+}\right)<\infty$. Suppose that $q$ and $\bar{q}$ are constants satisfying $1<q, \bar{q}<\infty$. Suppose also that $w$ and $v$ are weight functions on $\mathbb{R}_{+}$and that $T$ is an admissible operator on $\mathbb{R}_{+}$.

Then the inequality

$$
\|v T f\|_{\left(L^{p \cdot(\cdot)}\left(\mathbb{R}_{+}\right), l q\right)_{d}} \leq c\|w f\|_{\left(L^{\bar{p} \cdot(\cdot)}\left(\mathbb{R}_{+}\right), l \bar{q}\right)_{d}}
$$

holds if

(i) $T^{d}$ is bounded from $l^{\bar{q}}\left(\left\{\bar{w}_{n}\right\}\right)$ to $l^{q}\left(\left\{\bar{v}_{n}\right\}\right)$ where $\bar{w}_{n}:=\left\|\chi_{\left(2^{n-1}, 2^{n}\right)}(\cdot) w^{-1}(\cdot)\right\|_{L^{\prime}(\cdot)}^{-\bar{q}}$, $\bar{v}_{n}:=\left\|\chi_{\left(2^{n}, 2^{n+1}\right)}(\cdot) v(\cdot)\right\|_{L^{p(\cdot)}}^{q}$.

(ii) (a) $\sup _{n \in \mathbb{Z}}\left\|T_{n}\right\|_{\left[L_{w^{p}(\cdot) \cdot(\cdot)}^{\left.\bar{p}\left(2^{n-1}, 2^{n+2}\right) \rightarrow L_{v^{p(\cdot)}}^{p(\cdot)}\left(2^{n-1}, 2^{n+2}\right)\right]}\right.}<\infty$ for $1<\bar{q} \leq q<\infty$.

(b) $\left\|T_{n}\right\|_{\left[L_{w, \bar{p}(\cdot)}^{\bar{p}(\cdot)}\left(2^{n-1}, 2^{n+2}\right) \rightarrow L_{\nu p(\cdot)}^{p(\cdot)}\left(2^{n-1}, 2^{n+2}\right)\right]} \in l^{s}$ with $\frac{1}{s}=\frac{1}{q}-\frac{1}{\bar{q}}$ for $1<q<\bar{q}<\infty$.

Conversely, if (3.3) holds, then

(1) conditions (ii) are satisfied;

(2) condition (i) is also satisfied but for $w \equiv$ const or for $p$ and $\bar{p}$ satisfying the condition $p \equiv$ const, $\bar{p} \equiv$ const outside some large interval $\left[0,2^{m_{0}}\right], m_{0} \in \mathbb{Z}$.

Proposition 3.2 gives criteria for the boundedness of $H_{v, w}$ in dyadic amalgams on $\mathbb{R}_{+}$but by the next statement we prove the two-weight inequality under slightly different conditions.

Proposition 3.3 Let $I:=\mathbb{R}_{+}$and let $1<\bar{p}_{-}(I) \leq \bar{p}(\cdot) \leq p(\cdot) \leq p_{+}(I)<\infty$. Let $1<\bar{q}, q<\infty$. Suppose that $p, \bar{p} \in \mathrm{WL}\left(\mathbb{R}_{+}\right)$and that $p \equiv p_{c} \equiv$ const outside some large interval $(0, b)$. Then the inequality

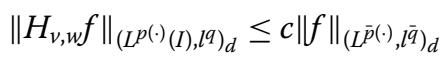

with a positive constant independent of $f$ holds if

(i) in the case $1<\bar{q} \leq q<\infty$,

(a)

$$
\sup _{m \in \mathbb{Z}}\left\{\sum_{n=m}^{\infty}\left\|\chi_{\left[2^{n}, 2^{n+1}\right)(\cdot)} v(\cdot)\right\|_{L^{p(\cdot)}}^{q}\right\}^{1 / q}\left\{\sum_{n=-\infty}^{m}\left\|\chi_{\left[2^{n-1}, 2^{n}\right)(\cdot)} w(\cdot)\right\|_{L^{p^{\prime} \cdot(\cdot)}}^{\bar{q}^{\prime}}\right\}^{1 / \bar{q}^{\prime}}<\infty,
$$

(b)

$$
\sup _{n \in \mathbb{Z} 0<\alpha<1} \sup _{0}\left\|\chi_{\left[2^{n+\alpha}, 2^{n+1}\right)(\cdot)} v(\cdot)\right\|_{L^{p(\cdot)}}\left\|w(\cdot) \chi_{\left(2^{n}, 2^{n+\alpha}\right) \cdot(\cdot)}\right\|_{L^{p^{\prime}(\cdot)}}<\infty ;
$$

(ii) in the case $1<q<\bar{q}<\infty$,

(a) $\left\{C_{n}\right\} \in l^{s}$, where

$$
C_{n}=\sup _{\beta \in(0,1)}\left\|\chi_{\left[2^{n+\beta}, 2^{n+1}\right)} v(\cdot)\right\|_{L^{p(\cdot)}}\left\|w(\cdot) \chi_{\left[2^{n}, 2^{n+\beta}\right)}\right\|_{L^{\bar{p}(\cdot)}},
$$


(b)

$$
\begin{aligned}
& \left\{\sum_{n \in \mathbb{Z}}\left(\sum_{k=n}^{\infty}\left\|\chi_{\left[2^{k}, 2^{k+1}\right)} v(\cdot)\right\|_{L^{p(\cdot)}}^{q}\right)^{s / q}\left(\sum_{k=-\infty}^{n}\left\|\chi_{\left[2^{k-1}, 2^{k}\right)}(\cdot) w(\cdot)\right\|_{L^{p^{\prime}} \cdot(\cdot)}^{1-\bar{q}^{\prime}}\right)^{s / \bar{q}^{\prime}}\right. \\
& \left.\quad \times\left\|\chi_{\left[2^{n}, 2^{n+1}\right)} v(\cdot)\right\|_{L^{p(\cdot)}}^{q}\right\}^{1 / s}<\infty,
\end{aligned}
$$

where $\frac{1}{s}=\frac{1}{\bar{q}}-\frac{1}{q}$.

Proof Let $1<\bar{q} \leq q<\infty$. Suppose that $f \geq 0$. We represent:

$$
\begin{aligned}
\left(H_{v, w} f\right)(x) & =v(x) \int_{0}^{2^{n}} f(t) w(t) d t+v(x) \int_{2^{n}}^{x} f(t) w(t) d t \\
& =:\left(H_{v, w}^{(1)} f\right)(x)+\left(H_{v, w}^{(2)} f\right)(x), \quad x \in\left[2^{n}, 2^{n+1}\right] .
\end{aligned}
$$

We have

$$
\begin{aligned}
\left\|\left(H_{v, w} f\right) \chi_{\left[2^{n}, 2^{n+1}\right)}(\cdot)\right\|_{L^{p(\cdot)}} \leq & \left\|v(\cdot) \chi_{\left[2^{n}, 2^{n+1}\right)(\cdot)}\right\|_{L^{p \cdot(\cdot)}}\left(\int_{0}^{2^{n}} f(t) w(t) d t\right) \\
& +\left\|v(x) \int_{2^{n}}^{x} f(t) w(t) d t\right\|_{L^{p(\cdot)}\left(\left[2^{n}, 2^{n+1}\right)\right)} \\
= & : S_{1}^{(n)}+S_{2}^{(n)} .
\end{aligned}
$$

Let $a_{k}:=\int_{2^{k-1}}^{2^{k}} f w$. Then by the discrete Hardy inequality (see Lemma C) and Hölder's inequality with respect to the exponents $\bar{p}(\cdot)$ and $(\bar{p}(\cdot))^{\prime}$ we derive

$$
\begin{aligned}
& \left(\sum_{n \in \mathbb{Z}}\left(S_{1}^{(n)}\right)^{q}\right)^{1 / q}=\left[\sum_{n \in \mathbb{Z}}\left\|v(\cdot) \chi_{\left[2^{n}, 2^{n+1}\right)}(\cdot)\right\|_{\left.L^{p \cdot(}\right)}^{q}\left(\sum_{k=-\infty}^{n} \int_{2^{k-1}}^{2^{k}} f(t) w(t) d t\right)^{q}\right]^{1 / q} \\
& \leq c\left[\sum_{n \in \mathbb{Z}}\left(\int_{2^{n-1}}^{2^{n}} f(t) w(t) d t\right)^{\bar{q}}\left\|w(\cdot) \chi_{\left[2^{n-1}, 2^{n}\right)}(\cdot)\right\|_{L^{\bar{p}^{\prime}}(\cdot)}^{-\overline{\bar{p}^{\prime}}}\right]^{1 / q}
\end{aligned}
$$

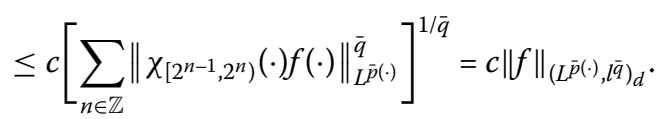

Further, by Corollary A and Theorem E, we have that

$$
\begin{aligned}
& \left(\sum_{n \in \mathbb{Z}}\left(S_{2}^{(n)}\right)^{q}\right)^{1 / q}=\left[\sum_{n \in \mathbb{Z}}\left\|v(x) \int_{2^{n}}^{x} f(t) w(t) d t\right\|_{L^{p(\cdot)}\left(2^{n}, 2^{n+1}\right)}^{q}\right]^{1 / q} \\
& \leq c\left[\sum_{n \in \mathbb{Z}}\left\|f(\cdot) \chi_{\left(2^{n}, 2^{n+1}\right)}(\cdot)\right\|_{L^{p}(\cdot)\left(2^{n}, 2^{n+1}\right)}^{q}\right]^{1 / q} \\
& \leq c\left[\sum_{n \in \mathbb{Z}}\left\|f(\cdot) \chi_{\left(2^{n}, 2^{n+1}\right)}(\cdot)\right\|_{L^{\bar{p}(\cdot)}\left(2^{n}, 2^{n+1}\right)}^{\bar{q}}\right]^{1 / \bar{q}}
\end{aligned}
$$

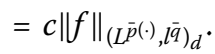


Let $1<q<\bar{q}<\infty$. Using representation (3.4), we derive

$$
\begin{aligned}
\left\|\left(H_{v, w} f\right)\right\|_{\left(L^{p(\cdot)}\left(\mathbb{R}_{+}\right), l q\right)} \leq & {\left[\sum_{n \in \mathbb{Z}}\left\|\chi_{\left[2^{n}, 2^{n+1}\right)} H_{v, w}^{(1)} f\right\|_{L^{p(\cdot)}}^{q}\right]^{1 / q} } \\
& +\left[\sum_{n \in \mathbb{Z}}\left\|\chi_{\left[2^{n}, 2^{n+1}\right)} H_{v, w}^{(2)} f\right\|_{L^{p(\cdot)}}^{q}\right]^{1 / q} \\
= & : S_{1}+S_{2} .
\end{aligned}
$$

We estimate $S_{1}$ and $S_{2}$.

$$
\begin{aligned}
S_{1} & =\left[\sum_{n \in \mathbb{Z}}\left\|\chi_{\left[2^{n}, 2^{n+1}\right)}(\cdot) v(\cdot)\right\|_{L^{p(\cdot)}}^{q}\left(\int_{0}^{2^{n}} f w\right)^{q}\right]^{1 / q} \\
& =\left[\sum_{n \in \mathbb{Z}}\left\|\chi_{\left[2^{n}, 2^{n+1}\right)}(\cdot) v(\cdot)\right\|_{L^{p(\cdot)}}^{q}\left(\sum_{k=-\infty}^{n} \int_{2^{k-1}}^{2^{k}} f w\right)^{q}\right]^{1 / q} .
\end{aligned}
$$

By the two-weight inequality for the discrete Hardy transform (see Lemma C), we have

$$
\begin{aligned}
S_{1} & \leq c\left[\sum_{n \in \mathbb{Z}}\left\|\chi_{\left[2^{n-1}, 2^{n)}\right)}(\cdot) w(\cdot)\right\|_{L^{\bar{p}^{\prime}(\cdot)}}^{-\bar{q}}\left(\int_{2^{n-1}}^{2^{n}} f w\right)^{\bar{q}}\right]^{1 / \bar{q}} \\
& \leq c\left[\sum_{n \in \mathbb{Z}}\left\|\chi_{\left[2^{n-1}, 2^{n}\right)}(\cdot) w(\cdot)\right\|_{L^{\bar{p}^{\prime}(\cdot)}}^{-\bar{q}}\left\|\chi_{\left[2^{n-1}, 2^{n}\right)} f\right\|_{L^{\bar{p}(\cdot)}}^{\bar{q}}\left\|\chi_{\left[2^{n-1}, 2^{n}\right)} w\right\|_{L_{\bar{p}^{\prime}}(\cdot)}^{\bar{q}}\right]^{1 / \bar{q}} \\
& \leq c\|f\|_{\left(L^{\bar{p} \cdot(\cdot)}\left(\mathbb{R}_{+}\right), l^{\bar{q}}\right)} \cdot
\end{aligned}
$$

Now we estimate $S_{2}$. Using Corollary A for intervals $\left(2^{n}, 2^{n+1}\right.$ ] and Hölder's inequality, we find that

$$
\begin{aligned}
S_{2} & \leq c\left\{\sum_{n \in \mathbb{Z}} C_{n}^{q}\left\|\chi_{\left[2^{n}, 2^{n+1}\right)} f\right\|_{L^{\bar{p} \cdot \cdot}}^{q}\right\}^{1 / q} \\
& \leq c\left\{\left(\sum_{n \in \mathbb{Z}}\left\|\chi_{\left[2^{n}, 2^{n+1}\right)} f\right\|_{L^{\bar{p} \cdot \cdot}}^{\bar{q}}\right)^{q / \bar{q}}\left(\sum_{n \in \mathbb{Z}} C_{n}^{\frac{q \bar{q}}{\bar{q}-q}}\right)^{\frac{\bar{q}-q}{q}}\right\}^{1 / q} \\
& \leq c\left(\sum_{n \in \mathbb{Z}} C_{n}^{s}\right)^{1 / s}\|f\|_{\left(L^{\bar{p} \cdot(\cdot)}\left(\mathbb{R}_{+}\right), l^{\bar{q}}\right) d^{\cdot}}
\end{aligned}
$$

\subsection{Kernel operators on amalgams $\left(L^{p(\cdot)}\left(\mathbb{R}_{+}\right), I^{q}\right)_{d}$ and $\left(L^{p(\cdot)}(\mathbb{R}), l^{q}\right)$}

The conditions of general-type statements (see Propositions 3.1 and 3.2) are not easily verifiable for general kernel operators as well as for some concrete fractional integral operators such as the Riemann-Liouville fractional integral transform with variable parameter. That is why we investigate mapping properties of general kernel operators independently from general-type statements.

Let

$$
\left(K_{v} f\right)(x)=v(x) \int_{0}^{x} f(t) k(x, t) d t, \quad x>0 .
$$

One of our aims is to characterize a class of weights $v$ governing the boundedness of $K_{v}$ from $\left(L^{\bar{p} \cdot(\cdot)}, l^{\bar{q}}\right)_{d}$ to $\left(L^{p(\cdot)}, l^{q}\right)_{d}$. 
We will use the notation:

$$
\begin{aligned}
& B_{1}:=\sup _{m \in \mathbb{Z}}\left[\sum_{n=m}^{\infty}\left\|\chi_{\left(2^{n}, 2^{n+1}\right]}(x) k\left(x, \frac{x}{2}\right) v(x)\right\|_{L^{p(\cdot)}}^{q}\right]^{1 / q}\left[\sum_{n=-\infty}^{m}\left\|\chi_{\left(2^{n-1}, 2^{n}\right]}\right\|_{L^{p^{\prime}(\cdot)}}^{\bar{q}^{\prime}}\right]^{1 / \bar{q}^{\prime}} ; \\
& B_{2}:=\sup _{n \in \mathbb{Z}} \sup _{0<\alpha<1}\left\|\chi_{\left(2^{n+\alpha}, 2^{n+1}\right]} k(x, x / 2) v(x)\right\|_{L^{p(\cdot)}}\left\|\chi_{\left(2^{n}, 2^{n+\alpha}\right]}\right\|_{L^{\bar{p}^{\prime}(\cdot)} .} .
\end{aligned}
$$

Theorem 3.1 Let $I:=\mathbb{R}_{+}, 1<\bar{p}_{-}(I) \leq \bar{p}(\cdot) \leq p(\cdot) \leq p_{+}(I)<\infty$ and let $\bar{p}, p \in \mathrm{WL}(I)$. Suppose that $\bar{q}$ and $q$ are constants such that $1<\bar{q} \leq q<\infty$. Let $p(x) \equiv p_{c} \equiv$ const and $\bar{p}(x) \equiv$ $\bar{p}_{c} \equiv$ const outside some large interval $\left(0,2^{m_{0}}\right), m_{0} \in \mathbb{Z}$. Let $k \in V(I) \cap V_{\bar{p}^{\prime}(\cdot)}(I)$. Then $K_{v}$ is bounded from $\left(L^{\bar{p}(\cdot)}(I), l^{\bar{q}}\right)_{d}$ to $\left(L^{p(\cdot)}(I), l^{q}\right)_{d}$ if and only if $B<\infty$, where $B=\max \left\{B_{1}, B_{2}\right\}$.

Proof Sufficiency. Using the representation:

$$
\begin{aligned}
\left(K_{v} f\right)(x) & =v(x) \int_{0}^{x / 2} k(x, t) f(t) d t+v(x) \int_{x / 2}^{x} k(x, t) f(t) d t \\
& =:\left(K_{v}^{(1)} f\right)(x)+\left(K_{v}^{(2)} f\right)(x)
\end{aligned}
$$

we have that

$$
\left\|K_{v} f\right\|_{\left(L^{\left.p(\cdot), l^{q}\right)_{d}}\right.} \leq\left\|K_{v}^{(1)} f\right\|_{\left(L^{\left.p(\cdot), l^{q}\right)_{d}}\right.}+\left\|K_{v}^{(2)} f\right\|_{\left(L^{\left.p(\cdot), l^{q}\right)_{d}}\right.} \cdot
$$

Further, taking Proposition 3.3 and the condition $k \in V(I)$ into account, we find that

$$
\begin{aligned}
\left\|K_{v}^{(1)} f\right\|_{\left(L^{p(\cdot)(I), l q)} d\right.} & \leq c\left\|v(x) k\left(x, \frac{x}{2}\right) \int_{0}^{x} f(t) d t\right\|_{\left(L^{p \cdot(\cdot), l q}\right)_{d}} \\
& \leq c B\|f\|_{\left(\bar{L}^{(\cdot)}(I), l q\right)} \cdot
\end{aligned}
$$

Now observe that by the condition $k \in V_{\bar{p}^{\prime}(\cdot)}(I)$, Proposition A and Lemma A we obtain

$$
\begin{aligned}
& \left\|K_{v}^{(2)} f\right\|_{\left(L^{p(\cdot)}\left(\left[0,2^{m_{0}}+1\right)\right), l^{\prime}\right) d} \\
& \leq\left[\sum_{k=-\infty}^{+\infty}\left\|\chi_{\left(2^{k}, 2^{k+1}\right]}(x) v(x)\left(\int_{x / 2}^{x} f(t) k(x, t) d t\right)\right\|_{L^{p(x)}}^{q}\right]^{1 / q} \\
& \leq\left[\sum_{k=-\infty}^{+\infty}\left\|\chi_{\left(2^{k}, 2^{k+1}\right]}(x) v(x)\right\| \chi_{(x / 2, x)}(\cdot) f(\cdot)\left\|_{L^{\bar{p}(\cdot)}}\right\| \chi_{(x / 2, x)} k(x, \cdot)\left\|_{L^{\bar{p}^{\prime} \cdot(\cdot)}}\right\|_{L^{p(x)}}^{q}\right]^{1 / q} \\
& \leq\left[\sum_{k=-\infty}^{+\infty}\left\|\chi_{\left(2^{k}, 2^{k+1}\right]}(x) v(x) x^{\frac{1}{\bar{p}^{\prime}(x)}} k(x, x / 2)\right\|_{L^{p(x)}}^{q}\left\|\chi_{\left(2^{k-1}, 2^{k+1}\right)}(\cdot) f(\cdot)\right\|_{L^{\bar{p}^{(\cdot)}}}^{q}\right]^{1 / q} \\
& \leq c\left[\sum_{k=-\infty}^{+\infty} 2^{k q /(\bar{p})^{\prime}\left(2^{k}\right)}\left\|\chi_{\left(2^{k}, 2^{k+1}\right]}(x) v(x) k(x, x / 2)\right\|_{L^{p(x)}}^{q}\left\|\chi_{\left(2^{k-1}, 2^{k+1}\right)}(\cdot) f(\cdot)\right\|_{L^{\bar{p}(\cdot)}}^{q}\right]^{1 / q} \\
& \leq c \bar{B}_{1}\left[\sum_{k=-\infty}^{+\infty}\left\|\chi_{\left(2^{k-1}, 2^{k}\right)}(\cdot) f(\cdot)\right\|_{L^{\bar{p}(\cdot)}}^{q}\right]^{1 / q}+c \bar{B}_{1}\left[\sum_{k=-\infty}^{+\infty}\left\|\chi_{\left(2^{k}, 2^{k+1}\right)}(\cdot) f(\cdot)\right\|_{L^{\bar{p} \cdot(\cdot)}}^{q}\right]^{1 / q}
\end{aligned}
$$

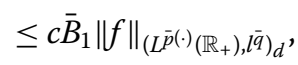


where

$$
\begin{aligned}
& \bar{B}_{1}:=\sup _{n \in \mathbb{Z}}\left\|\chi_{\left(2^{n}, 2^{n+1}\right]}(x) k\left(x, \frac{x}{2}\right) v(x)\right\|_{L^{p(x)}} 2^{1 /\left(\bar{p}_{n}\right)^{\prime}}, \\
& \bar{p}_{n}:= \begin{cases}\bar{p}\left(2^{n}\right), & n \leq m_{0}, \\
\bar{p}_{c}, & n>m_{0} .\end{cases}
\end{aligned}
$$

Let us now observe that by Proposition $\mathrm{A}$ and Lemma $\mathrm{A}, \bar{B}_{1} \approx \bar{A} \leq c B_{1}$, where

$$
\bar{A}:=\sup _{k \in \mathbb{Z}}\left\|v(\cdot) k(x, x / 2) \chi_{\left(2^{k}, 2^{k+1}\right]}\right\|_{L^{p \cdot(\cdot)}}\left\|\chi_{\left(2^{\left.k-1,2^{k}\right]}\right.}(\cdot)\right\|_{L^{p^{\prime}(\cdot)}} .
$$

Necessity. Let $\bar{p}_{n}$ be the sequence defined above. Considering the test function $f_{n}=$ $\chi_{\left(2^{n}, 2^{n+1}\right]} 2^{-n / \bar{p}_{n}}$ in the boundedness of $K_{v}$ from $\left(L^{\bar{p}(\cdot)}(I), l^{\bar{q}}\right)_{d}$ to $\left(L^{p(\cdot)}(I), l^{q}\right)_{d}$ and taking the condition $k \in V(I)$ into account we have that

$$
I_{n}:=\left\|\chi_{\left(2^{n}, 2^{n+1}\right]}(x) v(x) k(x, x / 2)\right\|_{L^{p(x)}} \leq c 2^{-n /\left(\bar{p}_{n}\right)^{\prime}} .
$$

It is easy to see that

(i)

$$
\sum_{n=m}^{\infty} I_{n} \leq c\left(2^{-m / \bar{p}^{\prime}(0)}+2^{-m_{0} / \bar{p}_{c}^{\prime}}\right)
$$

for $m \leq m_{0}$;

(ii)

$$
\sum_{n=m}^{\infty} I_{n} \leq c 2^{-m_{0} / \bar{p}_{c}^{\prime}}
$$

for $m \geq m_{0}+1$.

Denoting $S_{m}:=\left[\sum_{n=m}^{\infty} I_{n}^{q}\right]^{1 / q}\left[\sum_{n=-\infty}^{m-1}\left\|\chi_{\left(2^{n}, 2^{n+1}\right]}\right\|_{L^{\bar{p}^{\prime}(\cdot)}}^{\bar{q}}\right]^{1 / \bar{q}}$ and taking (3.9), Proposition A and Lemma A into account we have for $m \leq m_{0}$,

$$
\begin{aligned}
S_{m} & \leq\left[\sum_{n=m}^{\infty} I_{n}^{q}\right]^{1 / q} 2^{m / \bar{p}^{\prime}(0)} \leq\left[2^{-m / \bar{p}^{\prime}(0)}+2^{-m_{0} / \bar{p}_{c}^{\prime}}\right] 2^{m / \bar{p}^{\prime}(0)} \\
& \leq 1+2^{m / \bar{p}^{\prime}(0)} 2^{-m_{0} / \bar{p}_{c}^{\prime}} \leq 1+2^{m_{0} / \bar{p}^{\prime}(0)} 2^{-m_{0} / \bar{p}_{c}^{\prime}}<\infty .
\end{aligned}
$$

Similarly if $m \geq m_{0}+1$, then by (3.10),

$$
\begin{aligned}
S_{m} & \leq\left[\sum_{n=m}^{\infty} I_{n}^{q}\right]^{1 / q}\left[2^{m_{0} / \bar{p}^{\prime}(0)}+2^{m / \bar{p}_{c}^{\prime}}\right] \leq 2^{-m / \bar{p}_{c}^{\prime}}\left[2^{m_{0} / \bar{p}^{\prime}(0)}+2^{m / \bar{p}_{c}^{\prime}}\right] \\
& \leq 1+2^{m_{0} / \bar{p}^{\prime}(0)} 2^{-m_{0} / \bar{p}_{c}^{\prime}}<\infty .
\end{aligned}
$$

Hence, $B_{1}<\infty$. 
Let now $f$ be a function supported in $\left(2^{m}, 2^{m+1}\right]$. Then due to the boundedness of $K_{v}$ from $\left(L^{\bar{p}(\cdot)}(I), l^{\bar{q}}\right)_{d}$ to $\left(L^{p(\cdot)}(I), l^{q}\right)_{d}$ and the condition $k \in V(I)$ we have that

$$
\left\|\chi_{\left(2^{m}, 2^{m+1}\right]} v(x) k(x, x / 2)\left(\int_{2^{m}}^{x} f(y) d y\right)\right\|_{\left(L^{p(\cdot)(I), l q)} d\right.} \leq c\left\|\chi_{\left(2^{m}, 2^{m+1} f\right.} f\right\|_{\left(L^{(\cdot)}(I), l^{\bar{l}}\right) d^{\prime}},
$$

where the positive constant $c$ does not depend on $n$. Using Theorem B with respect to the intervals $\left[2^{m}, 2^{m+1}\right)$ and the weight pair $(\bar{v}, w)$, where $\bar{v}(x)=v(x) k(x, x / 2) \chi_{\left(2^{m}, 2^{m+1}\right]}$ and $\bar{w} \equiv$ const, it follows that $B_{2}<\infty$.

Remark 3.1 We have noticed in the proof of Theorem 3.1 that $B_{1} \approx \bar{A}$, where $\bar{A}$ is defined in the same proof.

Now we formulate the boundedness criteria for the kernel operator

$$
\left(\mathcal{K}_{v} f\right)=v(x) \int_{-\infty}^{x} k(x, t) f(t) d t, \quad x \in \mathbb{R}
$$

on amalgams defined on $\mathbb{R}$.

Let $k(x, y)$ be a kernel on $\{(x, y): y<x\}$ and $v, p, \bar{p}$ be defined on $\mathbb{R}$. For the next statement we define $\tilde{k}, \tilde{v}, p_{0}$ and $\bar{p}_{0}$ as follows:

$$
\begin{aligned}
& \tilde{k}(x, t):=\left(\frac{t^{-1 / \bar{p}^{\prime}\left(\log _{2} t\right)}}{x^{1 / p\left(\log _{2} x\right)}}\right) k\left(\log _{2} x, \log _{2} t\right), \\
& \tilde{v}(x):=v\left(\log _{2} x\right), \\
& \bar{p}_{0}(x):=\bar{p}\left(\log _{2} x\right), \quad p_{0}(x):=p\left(\log _{2} x\right) .
\end{aligned}
$$

Theorem 3.2 Let $1<\bar{p}_{-}(\mathbb{R}) \leq \bar{p}(x) \leq p(x) \leq p_{+}(\mathbb{R})<\infty$ and let $\bar{p}_{0}, p_{0} \in \mathrm{WL}\left(\mathbb{R}_{+}\right)$. Let $\bar{q}$ and $q$ are constants such that $1<\bar{q} \leq q<\infty$. Assume that $\bar{p}(x) \equiv \bar{p}_{c} \equiv$ const and $p(x) \equiv p_{c} \equiv$ const outside some large interval $(-\infty, b)$. Let $\tilde{k} \in V\left(\mathbb{R}_{+}\right) \cap V_{\left(\bar{p}_{0}(\cdot)\right)^{\prime}}\left(\mathbb{R}_{+}\right)$. Then $\mathcal{K}_{v}$ is bounded from $\left(L^{\bar{p}(\cdot)}(\mathbb{R}), l^{\bar{q}}\right)$ to $\left(L^{p(\cdot)}(\mathbb{R}), l^{q}\right)$ if and only if

$$
\begin{aligned}
& D_{1}:=\sup _{m \in \mathbb{Z}}\left[\sum_{n=m}^{\infty}\left\|\chi_{\left[2^{n}, 2^{n+1}\right)}(x) \tilde{k}\left(x, \frac{x}{2}\right) \tilde{v}(x)\right\|_{L^{p_{0}(\cdot)}\left(\mathbb{R}_{+}\right)}^{q}\right]^{1 / q} \\
& \times\left[\sum_{n=-\infty}^{m}\left\|\chi_{\left[2^{n-1}, 2^{n}\right)}\right\|_{L^{\left(\bar{p}_{0}(\cdot)\right)^{\prime}}\left(\mathbb{R}_{+}\right)}^{\bar{q}^{\prime}}\right]^{1 / \bar{q}^{\prime}}<\infty, \\
& D_{2}:=\sup _{n \in \mathbb{Z}} \sup _{0<\alpha<1}\left\|\chi_{\left[2^{n+\alpha}, 2^{n+1}\right)} \tilde{k}\left(x, \frac{x}{2}\right) \tilde{v}(x)\right\|_{L^{p_{0}(\cdot)\left(\mathbb{R}_{+}\right)}}\left\|\chi_{\left[2^{n}, 2^{n+\alpha}\right)}\right\|_{L^{\left(\bar{p}_{0}(\cdot)\right)^{\prime}\left(\mathbb{R}_{+}\right)}}<\infty \text {. }
\end{aligned}
$$

Proof The proof follows from Theorem 3.1 by the change of variable $z \rightarrow \log _{2} t$.

Let

$$
\left(\mathcal{R}_{\alpha(\cdot)} f\right)(x)=v(x) \int_{-\infty}^{x} \frac{2^{t} f(t)}{(x-t)^{1-\alpha(x)}} d t,
$$

where $0<\inf \alpha \leq \sup \alpha<1$ and $x \in \mathbb{R}_{+}$.

By virtue of Theorem 3.2 and Example 2.1 we can easily deduce the next statement. 
Corollary 3.1 Let $p, \bar{p}, q$ and $\bar{q}$ be constants. Suppose that $\alpha$ is a measurable function on $\mathbb{R}$ and that $1<\bar{p} \leq p<\infty, 1<\bar{q} \leq q<\infty, \frac{1}{\bar{p}}<\alpha(x)<1$. Then the operator $\mathcal{R}_{\alpha(\cdot)}$ is bounded from $\left(L^{\bar{p}}, l^{\bar{q}}\right)$ to $\left(L^{p}, l^{q}\right)$ if and only if

$$
\begin{aligned}
& \tilde{D}_{1}=\sup _{m \in \mathbb{Z}}\left[\sum_{n=m}^{\infty}\left(\int_{n}^{n+1}\left(2^{u}\right)^{\frac{p}{\bar{p}}} \nu^{p}(u) d u\right)^{q / p}\right]^{1 / q} 2^{m / \bar{p}^{\prime}}<\infty, \\
& \tilde{D}_{2}=\sup _{n \in \mathbb{Z}} \sup _{0<\beta<1}\left(\int_{n+\beta}^{n+1}\left(2^{u}\right)^{\frac{p}{\bar{p}}} \nu^{p}(u) d u\right)^{1 / p}\left(2^{n}\left(2^{\beta}-1\right)\right)^{1 / \bar{p}^{\prime}}<\infty .
\end{aligned}
$$

Moreover, there are positive constants $c_{1}$ and $c_{2}$ depending on $p, \bar{p}, q, \bar{q}$ and $\alpha$ such that $c_{1} \max \left\{\tilde{D}_{1}, \tilde{D}_{2}\right\} \leq\left\|\mathcal{R}_{\alpha(\cdot)}\right\| \leq c_{2} \max \left\{\tilde{D}_{1}, \tilde{D}_{2}\right\}$.

\section{Compactness of kernel operators on VEAS}

In this section, we derive compactness necessary and sufficient conditions for kernel operators on VEAS. Since for the amalgam norm we have the property $\left\|f_{n}\right\|_{\left(L^{p \cdot(\cdot)}(I), l^{q}\right)_{\alpha}} \downarrow 0$ when $f_{n} \downarrow 0$ a.e. $\left(f_{n} \in\left(L^{p(\cdot)}(I), l^{q}\right)_{\alpha}\right)$, therefore, the following statement holds (see [46], Chap. XI).

Proposition 4.1 Let $p, \bar{p}$ be measurable functions on I such that $1<\bar{p}, p<\infty$. Let $q, \bar{q}$ be constants satisfying the condition $1<q, \bar{q}<\infty$. Then the set of all functions of the form

$$
k_{n}(s, t) \equiv \sum_{i=1}^{n} \eta_{i}(s) \lambda_{i}(t), \quad s, t \in I
$$

is dense in the mixed norm space $\left(L^{p(\cdot)}(I), l^{q}\right)_{\alpha}\left[\left(L^{\bar{p}(\cdot)}(I), l^{\bar{q}}\right)_{\alpha}\right]$, where $\lambda_{i} \equiv \chi_{B_{i}}, \chi_{B_{i}} \in\left(L^{\bar{p}(\cdot)}(I)\right.$, $\left.l^{\bar{q}}\right)_{\alpha}\left(B_{i}\right.$ are measurable disjoint sets of $\left.I\right)$ and $\eta_{i} \in\left(L^{p(\cdot)}(I), l^{q}\right)_{\alpha} \cap L^{\infty}(I)$.

The next statement gives sufficient condition for the kernel operator to be compact on amalgams defined on $\mathbb{R}_{+}$.

Proposition 4.2 Let $p(x)$ and $q(x)$ be measurable functions on an interval $I \subseteq \mathbb{R}_{+}$. Suppose that $1<p_{-}(I) \leq p_{+}(I)<\infty, 1<\bar{p}_{-}(I) \leq \bar{p}_{+}(I)<\infty$. Let $q, \bar{q}$ be constants such that $1<\bar{q}$, $q<\infty$. If

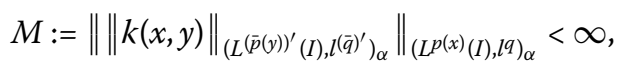

where $k$ is a non-negative kernel, then the operator

$$
K f(x)=\int_{I} k(x, y) f(y) d y
$$

is compact from $\left(L^{\bar{p}(\cdot)}(I), l^{\bar{q}}\right)_{\alpha}$ to $\left(L^{p(\cdot)}(I), l^{q}\right)_{\alpha}$.

Proof By Proposition 4.1 the set of functions

$$
k_{m}(s, t)=\sum_{i=1}^{m} \eta_{i}(s) \lambda_{i}(t), \quad s, t \in I,
$$


is dense in $\left(L^{p(\cdot)}(I), l^{q}\right)_{\alpha}\left[\left(L^{\bar{p}^{\prime}(\cdot)}(I), l^{\bar{q}^{\prime}}\right)_{\alpha}\right]$. By Hölder's inequality for amalgam spaces (see Theorem $\mathrm{D})$, we have

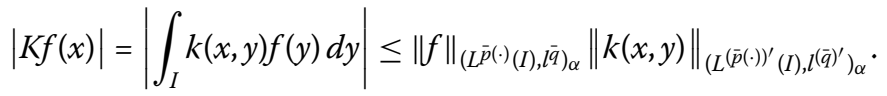

Hence,

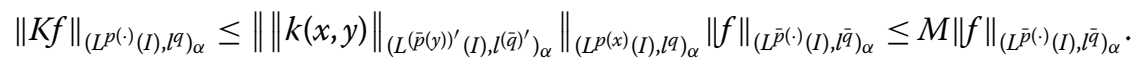

This means that $\|K\| \leq M$.

Now we prove the compactness of $K$. For each $n \in \mathbb{N}$, let

$$
\left(K_{n} \phi\right)(x)=\int_{I} k_{n}(x, y) \phi(y) d y .
$$

Note that

$$
\left(K_{n} \phi\right)(x)=\int_{I} k_{n}(x, y) \phi(y) d y=\sum_{i=1}^{n} \eta_{i}(x) \int_{I} \lambda_{i}(y) \phi(y) d y=: \sum_{i=1}^{n} \eta_{i}(x) b_{i},
$$

where

$$
b_{i}=\int_{I} \lambda_{i}(y) \phi(y) d y .
$$

This means that $K_{n}$ is a finite rank operator, i.e., it is compact. Further, let $\epsilon>0$. Using the above-mentioned arguments, we have that there is $N_{0} \in \mathbb{N}$ such that for $n>N_{0}$,

$$
\left\|K-K_{n}\right\| \leq\|\| k(x, y)-k_{n}(x, y)\left\|_{\left(L^{(\bar{p}(y))^{\prime}(I), l(\bar{p})^{\prime}}\right)_{\alpha}}\right\|_{\left(L^{p(x)}(I), l q\right)_{\alpha}}<\epsilon .
$$

Thus $K$ can be represented as a limit of finite rank operators. Hence, $K$ is compact.

Theorem 4.1 Let $1<\bar{p}_{-}\left(\mathbb{R}_{+}\right) \leq \bar{p}(x) \leq p(x) \leq p_{+}\left(\mathbb{R}_{+}\right)<\infty$ and let $\bar{p}, p \in \mathrm{WL}\left(\mathbb{R}_{+}\right)$. Let $\bar{q}$ and $q$ be constants such that $1<\bar{q} \leq q<\infty$. Assume that $k \in V\left(\mathbb{R}_{+}\right) \cap V_{(\bar{p}(\cdot))^{\prime}}\left(\mathbb{R}_{+}\right)$. Suppose that $\bar{p}(x) \equiv \bar{p}_{c} \equiv$ const and $p(x) \equiv p_{c} \equiv$ const outside some large interval $\left(0,2^{m_{0}}\right)$. Then $K_{v}$ is compact from $\left(L^{\bar{p}(\cdot)}, l^{\bar{q}}\right)_{d}$ to $\left(L^{p(\cdot)}, l^{q}\right)_{d}$ if and only if

$$
\begin{aligned}
\text { (i) } & B_{1}<\infty ; \quad B_{2}<\infty, \\
\text { (ii) } & \lim _{m \rightarrow-\infty} B_{1}(m)=\lim _{m \rightarrow+\infty} \mathbb{B}_{1}(m)=0, \\
\text { (iii) } & \lim _{n \rightarrow-\infty} B_{2}(n)=\lim _{n \rightarrow+\infty} B_{2}(n)=0,
\end{aligned}
$$

where, $B_{1}$ and $B_{2}$ are defined by (3.5) and (3.6), respectively, and

$$
\begin{aligned}
& B_{1}(m):=\left\|\chi_{\left[2^{m}, 2^{m+1}\right)} k(x, x / 2) v(x)\right\|_{L^{p \cdot(\cdot)}} 2^{m / \bar{p}^{\prime}(0)} ; \\
& \mathbb{B}_{1}(m):=\left[\sum_{n=m}^{\infty}\left\|\chi_{\left[2^{n}, 2^{n+1}\right)} k(x, x / 2) v(x)\right\|_{L^{p(\cdot)}}^{q}\right]^{1 / q}\left[\sum_{n=-\infty}^{m}\left\|\chi_{\left[2^{n-1}, 2^{n}\right)}(\cdot)\right\|_{L^{(\bar{q}(\cdot))^{\prime}}}^{(\bar{q})^{\prime}}\right]^{1 /(\bar{q})^{\prime}}, \\
& B_{2}(n):=\sup _{0<\alpha<1}\left\|\chi_{\left[2^{n+\alpha}, 2^{n+1}\right)}(x) v(x) k(x, x / 2)\right\|_{L^{p(\cdot)}}\left\|\chi_{\left(2^{n}, 2^{n+\alpha}\right)}(\cdot)\right\|_{L^{(\bar{p}(\cdot))^{\prime}} \cdot} .
\end{aligned}
$$


Proof Sufficiency. Let $k_{0}, n_{0}$ be integers such that $k_{0}<m_{0}<n_{0}$. Then we represent $K_{v}$ as follows:

$$
\begin{aligned}
\left(K_{v} f\right)(x)= & \chi_{\left[0,2^{k_{0}}\right]}(x) K_{v}\left(f \chi_{\left[0,2^{k_{0}}\right)}\right)(x)+\chi_{\left(2^{\left.k_{0}, 2^{n_{0}}\right)}\right.}(x) K_{v}\left(f \chi_{\left[0,2^{n_{0}}\right)}\right)(x) \\
& +\chi_{\left[2^{n_{0}}, \infty\right)}(x) K_{v}\left(f \chi_{\left[0,2^{n_{0}-1}\right)}\right)(x)+\chi_{\left[2^{n_{0}}, \infty\right)}(x) K_{v}\left(f \chi_{\left(2^{n_{0}-1}, \infty\right)}\right)(x) \\
=: & \left(K_{v}^{(1)} f\right)(x)+\left(K_{v}^{(2)} f\right)(x)+\left(K_{v}^{(3)} f\right)(x)+\left(K_{v}^{(4)} f\right)(x) .
\end{aligned}
$$

It is clear that

$$
\left(K_{v}^{(2)} f\right)(x)=\int_{\mathbb{R}_{+}} k_{2}(x, y) f(y) d y,
$$

where $k_{2}(x, y)=v(x) \chi_{\left(2^{\left.k_{0}, 2^{n}\right)}\right.}(x) k(x, y)$ if $y<x$ and $k_{2}(x, y)=0$ if $y \geq x$. Then

$$
\begin{aligned}
\| & \left\|k_{2}(x, y)\right\|_{\left(L^{(\bar{p})^{\prime}(y)}(I), l^{(\bar{q})^{\prime}}\right)_{d}} \|_{\left(L^{p(x)}\left(\left[^{k_{0}, 2^{m}}\right)\right), l q\right)_{d}} \\
& =\left\{\sum_{m=k_{0}}^{n_{0}-1}\left\|\chi_{\left(2^{m}, 2^{m+1}\right)}(x) v(x)\left(\sum_{n=-\infty}^{m}\left\|\chi_{\left(2^{n}, 2^{n+1}\right)} k(x, y)\right\|_{L^{(\bar{p})^{\prime}(y)}}^{(\bar{q})^{\prime}}\right)^{1 /(\bar{q})^{\prime}}\right\|_{L^{p(x)}}^{q}\right\}^{1 / q}=: J(x) .
\end{aligned}
$$

Denoting $I(x):=\sum_{n=-\infty}^{m}\left\|\chi_{\left(2^{n}, 2^{n+1}\right)^{n}} k(x, y)\right\|_{L^{(\bar{p})^{\prime}(y)}}^{(\bar{q})^{\prime}}, x \in\left[2^{m}, 2^{m+1}\right), k_{0} \leq m \leq n_{0}-1$, we represent $I(x)$ as

$$
\begin{aligned}
I(x)= & \sum_{n=-\infty}^{m-2}\left\|\chi_{\left(2^{n}, 2^{n+1}\right)}(y) k(x, y)\right\|_{L^{(\bar{p})^{\prime}(y)}}^{(\overline{\bar{q}})^{\prime}} \\
& +\left\|\chi_{\left(2^{m-1}, 2^{m}\right)}(y) k(x, y)\right\|_{L^{(\bar{p})^{\prime}(y)}}^{(\bar{q})^{\prime}}+\left\|\chi_{\left(2^{m}, x\right)}(y) k(x, y)\right\|_{L^{(\bar{p})^{\prime}(y)}}^{(\bar{q})^{\prime}} \\
=: & I_{1}(x)+I_{2}(x)+I_{3}(x) .
\end{aligned}
$$

Now we estimate $I_{1}(x), I_{2}(x)$ and $I_{3}(x)$ separately

$$
\begin{aligned}
I_{1}(x) & \leq c k^{(\bar{q})^{\prime}}\left(x, \frac{x}{2}\right) \sum_{n=-\infty}^{m-2}\left\|\chi_{\left[2^{n}, 2^{n}+1\right)}(y)\right\|_{L^{(\bar{p})^{\prime}(\cdot)}}^{(\bar{q})^{\prime}} \\
& \leq c k^{(\bar{q})^{\prime}}\left(x, \frac{x}{2}\right)\left[\sum_{n=-\infty}^{m_{0}}\left\|\chi_{\left[2^{n}, 2^{n}+1\right)}(\cdot)\right\|_{L^{(\bar{p})^{\prime}(\cdot)}}^{(\bar{q})^{\prime}}+\sum_{n=m_{0}+1}^{m-2}\left\|\chi_{\left[2^{n}, 2^{n}+1\right)}(y)\right\|_{L^{(\bar{p})^{\prime}(\bar{y})}}^{(\bar{q})^{\prime}}\right] \\
& \leq c k^{\overline{q^{\prime}}}\left(x, \frac{x}{2}\right)\left[\sum_{n=-\infty}^{m_{0}}\left(2^{n}\right)^{(\bar{q})^{\prime} /(\bar{p})^{\prime}(0)}+\sum_{m_{0}+1}^{n_{0}}\left(2^{n}\right)^{(\bar{q})^{\prime}(\overline{\bar{p}})_{c}^{\prime}}\right] \\
& \leq c k^{(\bar{q})^{\prime}}\left(x, \frac{x}{2}\right)\left[\left(2^{m_{0}}\right)^{(\bar{q})^{\prime}(\bar{p})^{\prime}(0)}+\left(2^{n_{0}}\right)^{(\bar{q})^{\prime}(\bar{p})^{\prime}}\right] .
\end{aligned}
$$

Further,

$$
\begin{aligned}
I_{2}(x)+I_{3}(x) & \leq 2\left\|\chi_{(0, x)} k(x, y)\right\|_{L^{(\bar{p})^{\prime}(y)}}^{(\bar{q})^{\prime}} \\
& \leq c\left\|\chi_{(0, x / 2)} k(x, y)\right\|_{L^{(\bar{p})^{\prime}(y)}}^{(\bar{q})^{\prime}}+c\left\|\chi_{(x / 2, x)} k(x, y)\right\|_{L^{(\bar{p})^{\prime}(y)}}^{(\bar{q})^{\prime}} \\
& \leq k^{(\bar{q})^{\prime}}\left(x, \frac{x}{2}\right)\left[\left\|\chi_{\left(0,2^{m}\right)}(y)\right\|_{L^{(\bar{p}(y)}}^{(\bar{q})^{\prime}}+x^{(\bar{q})^{\prime}(\bar{p})^{\prime}(x)}\right] .
\end{aligned}
$$


Considering separately the cases $m \leq m_{0}$ and $m>m_{0}$, by using Proposition A and Lemma A we find that

$$
I_{2}(x)+I_{3}(x) \leq c k^{(\bar{q})^{\prime}}\left(x, \frac{x}{2}\right)\left[\left(2^{m}\right)^{(\bar{q})^{\prime} /(\bar{p})^{\prime}(0)}+\left(2^{m}\right)^{(\bar{q})^{\prime} /(\bar{p})_{c}^{\prime}}\right]
$$

Consequently, since $k_{0} \leq m<n_{0}-1$, we have

$$
I(x) \leq c k^{(\bar{q})^{\prime}}\left(x, \frac{x}{2}\right)\left[\left(2^{n_{0}}\right)^{(\bar{q})^{\prime} /(\bar{p})^{\prime}(0)}+\left(2^{n_{0}}\right)^{(\bar{q})^{\prime} /(\bar{p})_{c}^{\prime}}\right]=: c k^{(\bar{q})^{\prime}}\left(x, \frac{x}{2}\right) B_{n_{0}} .
$$

Since $B_{1}<\infty$ we find that

$$
J(x) \leq B_{n_{0}}^{1 /(\bar{q})^{\prime}}\left[\sum_{m=k_{0}}^{n_{0}-1}\left\|\chi_{\left[2^{n}, 2^{n+1}\right)} k(x, x / 2) v(x)\right\|_{L^{p(\cdot)}}^{q}\right]^{1 / q}<\infty .
$$

So, by Proposition 4.2 , we conclude that $K_{v}^{(2)}$ is a compact operator. Further, write $K_{v}^{(3)}$ as follows:

$$
K_{v}^{(3)} f(x)=\int_{\mathbb{R}_{+}} k_{3}(x, y) f(y) d y
$$

where $k_{3}(x, y)=k(x, y) \chi_{\left(0,2^{n^{0}-1}\right)}(y) \chi_{\left[2^{n_{0}}, \infty\right)}(x) v(x)$ if $y<x$ and $k_{3}(x, y)=0$ if $y \geq x$. Then we have

$$
\begin{aligned}
\| & \left\|k_{3}(x, y)\right\|_{\left(L^{\left.(\bar{p})^{\prime}(y)(I), l(\bar{q})^{\prime}\right)}\right)_{d}} \|_{\left(L^{p(x)}(I), l^{q}\right)_{d}} \\
& =\left\{\sum_{m=n_{0}}^{\infty}\left\|\chi_{\left(2^{m}, 2^{m+1}\right)}(x) v(x)\left(\sum_{n=-\infty}^{n_{0}-2}\left\|\chi_{\left(2^{n}, 2^{n+1}\right)}(y) k(x, y)\right\|_{L^{(\bar{p})^{\prime}(y)}}^{(\overline{(})^{\prime}}\right)^{1 /(\bar{q})^{\prime}}\right\|_{L^{p(x)}}^{q}\right\}^{1 / q} \\
& \leq\left\{\sum_{m=n_{0}}^{\infty}\left\|\chi_{\left(2^{m}, 2^{m+1}\right)}(x) v(x) k(x, x / 2)\right\|_{L^{p(x)}}^{q}\right\}^{1 / q}\left(\sum_{n=-\infty}^{n_{0}-2}\left\|\chi_{\left(2^{n}, 2^{n+1}\right)}(y)\right\|_{L^{(\bar{p})^{\prime}(y)}}^{(\bar{q})^{\prime}}\right)^{1 /(\bar{q})^{\prime}} \\
& =: \text { G. }
\end{aligned}
$$

Denoting $F:=\left(\sum_{n=-\infty}^{n_{0}-1}\left\|\chi_{\left(2^{n}, 2^{n+1}\right)}(y)\right\|_{\left.L^{\bar{p}(\cdot)}\right)^{(\bar{q})^{\prime}}}^{1 /(\bar{q})^{\prime}}\right.$ and considering both cases when $m_{0} \leq n_{0}-2$ and $m_{0}>n_{0}-2$ separately, we derive as previously that

$$
F \leq c\left[\left(2^{m_{0}}\right)^{(\bar{q})^{\prime} /(\bar{p})^{\prime}(0)}+\left(2^{n_{0}}\right)^{(\bar{q})^{\prime} /(\bar{p})_{c}^{\prime}}\right]^{1 /(\bar{q})^{\prime}}=: B_{n_{0}, m_{0}}
$$

and since $B_{1}<\infty$ we have

$$
G \leq B_{n_{0}, m_{0}}\left[\sum_{m=n_{0}}^{\infty}\left\|\chi_{\left[2^{n}, 2^{n+1}\right)}(x) k(x, x / 2) v(x)\right\|_{L^{p(x)}}^{q}\right]^{1 / q}<\infty .
$$

Hence, by Proposition $4.2, K_{v}^{(3)}$ is compact.

Let us denote

$$
I_{m}:=\left\|\chi_{\left[2^{m}, 2^{m+1}\right)}(x) k(x, x / 2) v(x)\right\|_{L^{p(\cdot)}} .
$$


Following the proofs of Theorems 3.1, 3.2 and applying Proposition A and Lemma A, we have that

$$
\begin{aligned}
& \left\|K_{v}^{(1)}\right\|_{\left(L^{\bar{p}(\cdot)}(I), l^{\bar{q}}\right) \rightarrow\left(L^{(\cdot)}(I), l q\right)} l^{q} \\
& \leq \max \left\{\sup _{n \leq k_{0}}\left[\sum_{m=n}^{k_{0}} I_{m}^{q}\right]^{1 / q}\left[\sum_{m=-\infty}^{n}\left\|\chi_{\left[2^{m-1}, 2^{m}\right)}(\cdot)\right\|_{L^{(\bar{p}(\cdot))^{\prime}}}^{(\bar{q}]^{\prime}}\right]^{1 /(\bar{q})^{\prime}}, \sup _{m \leq k_{0}} B_{2}(m)\right\} \\
& \leq c \max \left\{\left[\sup _{m \leq k_{0}} I^{m} 2^{m / \bar{p}^{\prime}(0)}\right] \sup _{n \leq k_{0}}\left[\sum_{m=n}^{\infty} 2^{-m / \bar{p}^{\prime}(0)}\right]\left[\sum_{m=-\infty}^{n} 2^{m / \bar{p}^{\prime}(0)}\right], \sup _{m<k_{0}} B_{2}(m)\right\} \\
& \quad \leq c \max \left\{\sup _{m \leq k_{0}} I^{m} 2^{m / \bar{p}^{\prime}(0)}, \sup _{m<k_{0}} B_{2}(m)\right\} \\
& \rightarrow 0
\end{aligned}
$$

as $k_{0} \rightarrow 0$ because $\lim _{m \rightarrow-\infty} B_{1}(m)=\lim _{m \rightarrow-\infty} B_{2}(m)=0$. Further, applying Theorem 3.1, we find that

$$
\left\|K_{v}^{(4)}\right\|_{\left(L^{(\cdot)}(I), l^{\bar{q}}\right) \rightarrow\left(L^{p \cdot()}(I), l^{q}\right)} \leq \max \left\{\sup _{m \geq n_{0}} \mathbb{B}_{1}(m), \sup _{m \geq n_{0}} B_{2}(m)\right\} \rightarrow 0
$$

as $n_{0} \rightarrow+\infty$.

Hence,

$$
\left\|K_{v} f-K_{v}^{(2)} f-K_{v}^{(3)} f\right\| \leq\left\|K_{v}^{(1)} f\right\|+\left\|K_{v}^{(4)} f\right\| \rightarrow 0
$$

as $\mathbb{B}_{1}(m) \rightarrow 0, B_{i}(m) \rightarrow 0, i=1,2$. Hence $K_{v}$ is compact, since it is the limit of compact operators.

Necessity. First we show that $\lim _{m \rightarrow-\infty} B_{1}(m)=0$. Let $f_{n}=\chi_{\left(2^{n-1}, 2^{n+1}\right)} 2^{-n / \bar{p}_{n}}$, where $\bar{p}_{n}$ is defined in the proof of Theorem 3.1. Then $f_{n} \rightarrow 0$ weakly in $\left(L^{\bar{p}(\cdot)}(I), l^{\bar{q}}\right)_{d}$ as $n \rightarrow-\infty$. Indeed, let $\phi \in\left(L^{(\bar{p}(\cdot))^{\prime}}(I), l^{(\bar{q})^{\prime}}\right)_{d}$. Then

$$
\begin{aligned}
\left|\int_{0}^{\infty} f_{n}(y) \phi(y) d y\right| \leq & \left(\left\|\chi_{\left(2^{n-1}, 2^{n}\right]}\right\|_{L^{\bar{p}(\cdot)}}^{\overline{\bar{q}}}+\left\|\chi_{\left(2^{n}, 2^{n+1}\right]}\right\|_{L^{\bar{p}(\cdot)}}^{\overline{\bar{q}}}\right)^{1 / \bar{q}} 2^{-n / \bar{p}_{c}} \\
& \times\left(\left\|\phi \chi_{\left(2^{n-1}, 2^{n}\right]}\right\|_{L^{(\bar{p} \cdot(\cdot))^{\prime}}}^{\bar{q}}+\left\|\phi \chi_{\left(2^{n-1}, 2^{n]}\right]}\right\|_{L^{(\bar{p} \cdot(\cdot))^{\prime}}}\right)^{1 / \bar{q}} \\
\rightarrow & 0
\end{aligned}
$$

as $n \rightarrow-\infty$.

Observe now that

$$
\left\|K_{v} f_{n}\right\|_{\left(L^{p}(\cdot)(I), \bar{l}^{\bar{q}}\right)_{d}} \geq\left\|\chi_{\left(2^{n}, 2^{n+1)}\right.}(x) v(x) k(x, x / 2)\right\|_{L^{p(\cdot)}} 2^{n / \bar{p}_{n}^{\prime}}, \quad n \in \mathbb{Z} .
$$

Hence, $\lim _{n \rightarrow-\infty} B_{1}(n) \rightarrow 0$ because $K_{v}$ is compact and $\bar{p}_{n}=\bar{p}(0)$ if $n<m_{0}$.

Further, (4.2) implies that

$$
\left\|\chi_{\left(2^{n}, 2^{n+1}\right)}(x) v(x) k(x, x / 2)\right\|_{L^{p(\cdot)}} 2^{n /\left(\bar{p}_{c}\right)^{\prime}} \rightarrow 0
$$


To show that $\lim _{n \rightarrow+\infty} \mathbb{B}_{1}(n) \rightarrow 0$ we represent $\mathbb{B}_{1}(n)$ as follows:

$$
\begin{aligned}
\mathbb{B}_{1}(n) & =\left(\sum_{m=n}^{\infty} I_{m}^{q}\right)^{1 / q}\left(\sum_{m=-\infty}^{n-1}\left\|\chi_{\left(2^{m}, 2^{m+1}\right]}\right\|_{L^{(\bar{p} \cdot(\cdot))^{\prime}}}^{\bar{q}}\right)^{1 / \bar{q}} \\
& \leq\left(\sum_{m=n}^{\infty} I_{m}^{q}\right)^{1 / q}\left(\sum_{m=-\infty}^{m_{0}-1} 2^{m \bar{q} /(\bar{p}(0))^{\prime}}\right)^{1 / \bar{q}}+\left(\sum_{m=n}^{\infty} I_{m}^{q}\right)^{1 / q}\left(\sum_{m=m_{0}}^{n-1} 2^{m \bar{q} /\left(\bar{p}_{c}\right)^{\prime}}\right)^{1 / \bar{q}} \\
& =: J_{n}^{(1)}+J_{n}^{(2)},
\end{aligned}
$$

where $n \geq m_{0}$ and $I_{m}$ is defined by (4.1). Observe now that

$$
J_{n}^{(1)}=\left(\sum_{m=n}^{\infty} I_{m}^{q}\right)^{1 / q} 2^{m_{0} /(\bar{p}(0))^{\prime}} \rightarrow 0
$$

as $n \rightarrow+\infty$ because $\left(\sum_{m=n}^{\infty} I_{m}^{q}\right)^{1 / q} \rightarrow 0$ as $n \rightarrow+\infty$. The latter convergence follows from the convergence of the series.

Further,

$$
\begin{aligned}
J_{n}^{(2)} & \leq c \sup _{m \geq n}\left(I_{m} 2^{m /\left(\bar{p}_{c}\right)^{\prime}}\right) 2^{-n /\left(\bar{p}_{c}\right)^{\prime}} 2^{\left.n / \bar{p}_{c}\right)^{\prime}} \\
& \leq c \sup _{m \geq n} I_{m} 2^{m /\left(\bar{p}_{c}\right)^{\prime}} \rightarrow 0
\end{aligned}
$$

as $n \rightarrow+\infty$ because $I_{m} 2^{m /\left(\bar{p}_{c}\right)^{\prime}} \rightarrow 0$ as $m \rightarrow+\infty$ (see (4.2)). Hence, $\lim _{m \rightarrow+\infty} \mathbb{B}_{1}(m)=0$.

Further, it is easy to see that for $0<\alpha<1$ and $f_{n}$,

$$
\begin{aligned}
\left\|K_{v} f_{n}\right\|_{\left(L^{p(\cdot), l q)}\right)_{d}} & \geq 2^{-n / \bar{p}_{n}}\left\|\chi_{\left(2^{n}, 2^{n+1}\right)}(x) v(x) k(x, x / 2) x\right\|_{L^{p(\cdot)}} \\
& \geq 2^{n /\left(\bar{p}_{n}\right)^{\prime}}\left\|\chi_{\left(2^{n}, 2^{n+1}\right)}(x) v(x) k(x, x / 2)\right\|_{L^{p \cdot(\cdot)}} \\
& \geq c\left(2^{n}\left(2^{\alpha}-1\right)\right)^{1 /\left(\bar{p}_{n}\right)^{\prime}}\left\|\chi_{\left(2^{n+\alpha}, 2^{n+1}\right)}(x) v(x) k(x, x / 2)\right\|_{L^{p(\cdot)}} .
\end{aligned}
$$

Hence,

$$
\left\|K_{v} f_{n}\right\|_{\left(L^{\left.p(\cdot), l^{q}\right)_{d}} \geq\right.} \geq \sup _{0<\alpha<1}\left(2^{n}\left(2^{\alpha}-1\right)\right)^{1 /\left(\bar{p}_{n}\right)^{\prime}}\left\|\chi_{\left(2^{n+\alpha}, 2^{n+1}\right)}(x) v(x) k(x, x / 2)\right\|_{L^{p(\cdot)}} \rightarrow 0
$$

as $n \rightarrow+\infty$ or $n \rightarrow-\infty$.

The conditions $B_{1}<\infty$ and $B_{2}<\infty$ follow from the fact that every compact operator is bounded.

Now we formulate the compactness criteria for the kernel operator $\mathcal{K}_{v}$ defined on $\mathbb{R}$.

Theorem 4.2 Let $1<\bar{p}_{-}(\mathbb{R}) \leq \bar{p}(x) \leq p(x) \leq p_{+}(\mathbb{R})<\infty$ and let $\bar{p}_{0}, p_{0} \in \mathrm{WL}\left(\mathbb{R}_{+}\right)$. Let $\bar{q}$ and $q$ be constants such that $1<\bar{q} \leq q<\infty$. Assume that $\bar{p}(x) \equiv \bar{p}_{c} \equiv$ const and $p(x) \equiv$ $p_{c} \equiv$ const outside some large interval $\left(-\infty, 2^{m_{0}}\right)$. Let $\tilde{k} \in V\left(\mathbb{R}_{+}\right) \cap V_{\left(\bar{p}_{0}(\cdot)\right)^{\prime}}\left(\mathbb{R}_{+}\right)$. Then $\mathcal{K}_{v}$ is compact from $\left(L^{\bar{p}(\cdot)}, l^{\bar{q}}\right)$ to $\left(L^{p(\cdot)}, l^{q}\right)$ if and only if

$$
\text { (i) } \quad D_{1}=\sup _{m \in \mathbb{Z}} \mathbb{D}_{1}(m)<\infty ; \quad D_{2}=\sup _{n \in \mathbb{Z}} D_{2}(n)<\infty
$$


(ii)

$$
\lim _{m \rightarrow-\infty} D_{1}(m)=\lim _{m \rightarrow \infty} D_{1}(m)=0 \text {, }
$$

(iii)

$$
\lim _{n \rightarrow-\infty} D_{2}(n)=\lim _{n \rightarrow \infty} D_{2}(n)=0
$$

where

$$
\begin{aligned}
& D_{1}(m):=\left\|\chi_{\left[2^{m}, 2^{m+1}\right)} \tilde{k}(x, x / 2) \tilde{v}(x)\right\|_{L^{p_{0}(\cdot)}} 2^{m / \bar{p}_{0}^{\prime}(0)} ; \\
& \mathbb{D}_{1}(m):=\left[\sum_{n=m}^{\infty}\left\|\chi_{\left[2^{n}, 2^{n+1}\right)} \tilde{k}(x, x / 2) \tilde{v}(x)\right\|_{L^{p_{0}(\cdot)}}^{q}\right]^{1 / q} \\
& \times\left[\sum_{n=-\infty}^{m}\left\|\chi_{\left[2^{n-1}, 2^{n}\right)}(\cdot)\right\|_{L^{\bar{p}_{0}(\cdot)}}^{(\bar{q})^{\prime}}\right]^{1 /(\bar{q})^{\prime}} ; \\
& D_{2}(n):=\sup _{0<\alpha<1}\left\|\chi_{\left[2^{n+\alpha}, 2^{n+1}\right)}(x) \tilde{k}(x, x / 2) \tilde{v}(x)\right\|_{L^{p_{0}(\cdot)}}\left\|\chi_{\left(2^{n}, 2^{n+\alpha}\right)}(\cdot)\right\|_{L^{\left(\bar{p}_{0}(\cdot)\right)^{\prime}}} ;
\end{aligned}
$$

\section{$\tilde{k}, \tilde{v}$ and $p_{0}$ and $\bar{p}_{0}$ are defined in Section 3.}

Proof The proof follows from Theorem 4.1 by the change of variable $z \rightarrow \log _{2} t$.

\section{Competing interests}

The authors declare that they have no competing interests.

\section{Authors' contributions}

All authors carried out the proof. All authors conceived of the study, and participated in its design and coordination. All authors read and approved the final manuscript.

\section{Author details}

${ }^{1}$ Department of Mathematical Analysis, A. Razmadze Mathematical Institute, I. Javakhishvili Tbilisi State University, 2 University Str., Tbilisi, 0186, Georgia. ${ }^{2}$ International Black Sea University, 3 Agmashenebeli Ave., Tbilisi, 0131, Georgia. ${ }^{3}$ Department of Mathematics, Faculty of Informatics and Control Systems, Georgian Technical University, 77 Kostava Str., Tbilisi, Georgia. ${ }^{4}$ Abdus Salam School of Mathematical Sciences, GC University, 68-B New Muslim Town, Lahore, Pakistan.

\section{Acknowledgements}

The first and second authors were supported by the Shota Rustaveli National Science Foundation grant (Contract No. D/13-23). The part of this work is carried out at Abdus Salam School of Mathematical Sciences, GC University, Lahore. The second and third authors are thankful to the Higher Education Commission, Pakistan for the financial support. The authors are grateful to the anonymous referees for their remarks and suggestions.

\section{Received: 28 August 2012 Accepted: 1 April 2013 Published: 16 April 2013}

\section{References}

1. Edmunds, DE, Kokilashvili, V, Meskhi, A: On the boundedness and compactness of the weighted Hardy operators in $L^{p(x)}$ spaces. Georgian Math. J. 12(1), 27-44 (2005)

2. Kopaliani, TS: On some structural properties of Banach function spaces and boundedness of certain integral operators. Czechoslov. Math. J. 54(3), 791-805 (2004)

3. Cruz-Uribe, D, Mamedov, Fl: On a general weighted Hardy type inequality in the variable exponent Lebesgue spaces. Rev. Mat. Complut. 25(2), 335-367 (2012)

4. Samko, S: Convolution type operators in $L^{p(x)}\left(R^{n}\right)$. Integral Transforms Spec. Funct. 7(3-4), $261-284$ (1998)

5. Edmunds, DE, Meskhi, A: Potential-type operators in $L^{p(x)}$ spaces. Z. Anal. Anwend. 21, 681-690 (2002)

6. Diening, L: Riesz potentials and Sobolev embeddings on generalized Lebesgue and Sobolev spaces $L^{p(\cdot)}$ and $W^{k, p(\cdot)}$. Math. Nachr. 268, 31-43 (2004)

7. Capone, C, Cruz-Uribe, D, Fiorenza, A: The fractional maximal operator on variable $L^{p}$ spaces. Rev. Mat. Iberoam. 3(23), 747-770 (2007)

8. Cruz-Uribe, D, Fiorenza, A, Martell, JM, Perez, C: The boundedness of classical operators on variable $L^{p}$ spaces. Ann Acad. Sci. Fenn. Math. 31, 239-264 (2006)

9. Kokilashvili, V, Samko, S: Maximal and fractional operators in weighted $L^{p(x)}$ spaces. Rev. Mat. Iberoam. 20(2), 493-515 (2004)

10. Kokilashvili, $\bigvee$, Samko, S: On Sobolev theorem for Riesz-type potentials in Lebesgue spaces with variable exponent. Z. Anal. Anwend. 22(4), 899-910 (2003) 
11. Kokilashvili, V, Meskhi, A: Weighted criteria for generalized fractional maximal functions and potentials in Lebesgue spaces with variable exponent. Integral Transforms Spec. Funct. 18(9), 609-628 (2007)

12. Kokilashvili, V, Meskhi, A: Two-weight inequalities for fractional maximal functions and singular integrals in $L^{p(\cdot)}$ spaces. J. Math. Sci. 173(6), 656-673 (2011)

13. Kokilashvili, V, Meskhi, A, Sarwar, M: One and two weight estimates for one-sided operators in $L^{p(\cdot)}$ spaces. Eurasian Math. J. 1(1), 73-110 (2010)

14. Kokilashvili, V, Meskhi, A, Sarwar, M: Potential operators in variable exponent Lebesgue spaces: two-weight estimates J. Inequal. Appl. (2010). doi:10.1155/2010/329571

15. Meskhi, A: Measure of Non-Compactness for Integral Operators in Weighted Lebesgue Spaces. Nova Science Publishers, New York (2009)

16. Diening, L, Harjulehto, P, Hästö, P, Ružička, M: Lebesgue and Sobolev Spaces with Variable Exponents. Lecture Notes in Mathematics, vol. 2017. Springer, Berlin (2011)

17. Musielak, J: Orlicz Spaces and Modular Spaces. Lecture Notes in Mathematics, vol. 1034. Springer, Berlin (1983)

18. Musielak, J, Orlicz, W: On modular spaces. Stud. Math. 18, 49-65 (1959)

19. Nakano, H: Topology of Linear Topological Spaces. Moruzen Co. Ltd, Tokyo (1981)

20. Ružička, M: Electrorheological Fluids: Modeling and Mathematical Theory. Lecture Notes in Mathematics, vol. 1748. Springer, Berlin (2000)

21. Kokilashvili, V: On a progress in the theory of integral operators in weighted Banach function spaces. In: Function Spaces, Differential Operators and Nonlinear Analysis. Proceedings of the Conference held in Milovy, Bohemian-Moravian Uplands, May 28-June 2. Math. Inst. Acad. Sci. of Czech Republic, Prague (2004)

22. Kovácik, O, Rákosník, J: On spaces $L^{p(x)}$ and $W^{k, p(x)}$. Czechoslov. Math. J. 41(4), 592-618 (1991)

23. Samko, S: Convolution type operators in $L^{p(x)}$. Integral Transforms Spec. Funct. 7(1-2), 123-144 (1998)

24. Sharapudinov, II: The topology of the space $\mathcal{L}^{p(t)}([0,1])$. Mat. Zametki 26(4), 613-632 (1979) (Russian)

25. Diening, L: Maximal function on generalized Lebesgue spaces $L^{p(\cdot)}$. Math. Inequal. Appl. 7(2), 245-253 (2004)

26. Ashraf, $U$, Kokilashvili, V, Meskhi, A: Weight characterization of the trace inequality for the generalized Riemann-Liouville transform in $L^{p(x)}$ spaces. Math. Inequal. Appl. 13(1), 63-81 (2010)

27. Muckenhoupt, B: Hardy's inequality with weights. Stud. Math. 44, 31-38 (1972)

28. Kokilashvili, VM: On Hardy's inequalities in weighted spaces. Soobsch. Akad. Nauk Gruz. SSR 96, 37-40 (1979) (Russian)

29. Maz'ya, VG: Sobolev Spaces. Springer, Berlin (1985)

30. Kufner, A, Persson, LE: Weighted Inequalities of Hardy Type. World Scientific, River Edge (2003)

31. Cañestro, MIA, Salvador, PO: Boundedness of positive operators on weighted amalgams. J. Inequal. Appl. (2011). doi:10.1186/1029-242X-2011-13

32. Carton-Lebrun, C, Heinig, HP, Hofmann, SC: Integral operators on weighted amalgams. Stud. Math. 109(2), 133-175 (1994)

33. Meskhi, A: Criteria for the boundedness and compactness of integral transforms with positive kernels. Proc. Edinb. Math. Soc. 44(2), 267-284 (2001)

34. Edmunds, DE, Kokilashvili, V, Meskhi, A: Bounded and Compact Integral Operators. Kluwer Academic, Dordrecht (2002)

35. Stewart, J, Watson, S: Irregular amalgams. Int. J. Math. Math. Sci. 9(2), 331-340 (1986)

36. Wiener, N: On the representation of functions by trigonometrical integrals. Math. Z. 24, 575-616 (1926)

37. Wiener, N: Tauberian theorem. Ann. Math. 33, 1-100 (1932)

38. Jakimovski, A, Russell, DC: Interpolation by functions with $m$ th derivative in pre-assigned spaces. In: Cheney, EW (ed.) Approximation Theory III, Conference Proc. Texas, 1980, pp. 531-536. Academic Press, New York (1980)

39. Day, MM: Some more uniformly convex spaces. Bull. Am. Math. Soc. 47, 504-507 (1941)

40. Köthe, G: Topological Vector Spaces, vol. I. Springer, New York (1969)

41. Fournier, JF, Stewart, J: Amalgams of $L^{p}$ and 19 . Bull. Am. Math. Soc. 13(1), 1-21 (1985)

42. Edmunds, DE, Fiorenza, A, Meskhi, A: On the measure of non-compactness for some classical operators. Acta Math. $\operatorname{Sin} .22(6), 1847-1862(2006)$

43. Salvador, PO, Ramírez Torreblanca, C: Hardy operators on weighted amalgams. Proc. R. Soc. Edinb. A 140(1), 175-188 (2010)

44. Heinig, HP, Kufner, A: Weighted Friedrichs inequalities in amalgams. Czechoslov. Math. J. 43(118)(2), 285-308 (1993)

45. Cañestro, MIA, Salvador, PO: Boundedness of generalized Hardy operators on weighted amalgam spaces. Math. Inequal. Appl. 13(2), 305-318 (2010)

46. Kantorovich, LP, Akilov, GP: Functional Analysis. Pergamon, Oxford (1982)

doi:10.1186/1029-242X-2013-173

Cite this article as: Kokilashvili et al.: Weighted kernel operators in variable exponent amalgam spaces. Journal of Inequalities and Applications 2013 2013:173. 